La Revue

des Droits

de l'Homme

\section{La Revue des droits de l'homme}

Revue du Centre de recherches et d'études sur les droits fondamentaux

$3 \mid 2013$

Revue des droits de l'homme $-\mathrm{N}^{\circ} 3$

\title{
Le rôle du conseil dans le cadre des témoignages incriminant leur auteur
}

Le cas de la Cour pénale internationale

\section{Ghislain Mabanga}

\section{(2) OpenEdition}

Journals

Édition électronique

URL : http://journals.openedition.org/revdh/237

DOI : $10.4000 /$ revdh.237

ISSN : 2264-119X

Éditeur

Centre de recherches et d'études sur les droits fondamentaux

Référence électronique

Ghislain Mabanga, "Le rôle du conseil dans le cadre des témoignages incriminant leur auteur », La Revue des droits de l'homme [En ligne], 3 | 2013, mis en ligne le 07 novembre 2017, consulté le 08 juillet 2020. URL : http://journals.openedition.org/revdh/237 ; DOI : https://doi.org/10.4000/revdh.237

Ce document a été généré automatiquement le 8 juillet 2020.

Tous droits réservés 


\section{Le rôle du conseil dans le cadre des témoignages incriminant leur auteur}

Le cas de la Cour pénale internationale

Ghislain Mabanga

\section{NOTE DE L'ÉDITEUR}

Cet article fait suite à une communication lors du $9^{\mathrm{e}}$ séminaire des conseils de la Cour pénale internationale tenu à La Haye du 16 au 17 mai 2011. Il a été actualisé à l'occasion d'une intervention lors d'une conférence du 20 décembre 2012 sur le thème «Avocats et justice pénale internationale, le cas de la Cour pénale internationale », organisée dans le cadre du cours de "Droit pénal, aspects internationaux ", dispensé aux étudiants de Master 1 de l'Université Paris Ouest - Nanterre La Défense par Mmes Marina Eudes et Florence Bellivier.

\section{Introduction}

1 La place de la preuve testimoniale dans le procès pénal international.- S'il est un élément essentiel qui singularise le procès pénal international par rapport au procès international $^{1}$ en général, c'est la preuve testimoniale qui en constitue la pierre angulaire $^{2}$. En effet, ainsi que le relève, avec à propos, le Professeur Kaï Ambos, «international criminal trials for war crimes and crimes against humanity strongly depend on witness testimony $\|^{3}$. Il n'est, dès lors, pas étonnant que le premier procès pénal international, tenu par le Tribunal militaire international de Nuremberg, qui se déroula du 20 novembre 1945 au $1^{\text {er }}$ octobre 1946, vît défiler, hormis les 19 accusés, pas moins de 33 témoins à charge et 66 à décharge ${ }^{4}$. En 204 jours d'audiences, le premier procès de la Cour pénale internationale (ci-après «la CPI » ou «la Cour »), qui s'ouvrit le 26 janvier 2009, vit, lui, déposer 67 témoins, dont 36 de l’Accusation, et 24 de la Défense ${ }^{5}$. 
2 La protection subséquente des témoins.- L'on notera, cependant, que la place centrale qu'ont toujours occupée les témoins dans le procès pénal international contraste étrangement avec l'absence de mécanismes appropriés permettant d'assurer leur protection. Il fallut, en effet, attendre 48 ans après le procès de Nuremberg pour voir apparaître, à l'occasion de la création du Tribunal pénal international pour l'exYougoslavie (ci-après "TPIY») ${ }^{6}$, une structure spécifique destinée à assurer la protection des victimes et des témoins, appelée la section d'aide aux victimes et aux témoins ${ }^{7}$. Une structure identique fut mise sur pied l'année suivante pour le Tribunal pénal international pour le Rwanda (ci-après «TPIR ») ${ }^{8}$. Créée le 17 juillet $1998^{9}$, la CPI demeura dans la même logique en prévoyant, au sein de son Greffe, une Division d'aide aux victimes et aux témoins chargée "de conseiller et d'aider de toute manière appropriée les témoins, les victimes qui comparaissent devant la Cour et les autres personnes auxquelles les dépositions de ces témoins peuvent faire courir un risque $»^{10}$.

3 La protection particulière des témoins susceptibles d'auto-incrimination.- L'on ne pourrait, cependant, ne pas constater qu'aucune de ces structures n'a prévu de mesures appropriées à l'égard d'une catégorie particulière de témoins. Il s'agit de ceux qui sont plus ou moins impliqués dans les faits dont est saisi le juge pénal international et qui, par voie de conséquence, encourent des poursuites du fait de leurs dépositions respectives. Certes, nombre de règlements de procédure et de preuve (ci-après « RPP ») des juridictions pénales internationales (ci-après «JPI ») organisent des mécanismes de protection des témoins contre le risque d'auto-incrimination ${ }^{11}$. Néanmoins, aucun d'entre eux n'est allé jusqu'à considérer ces témoins comme des suspects ou accusés potentiels et, en conséquence, à leur reconnaître le droit au conseil reconnu à tous les suspects ${ }^{12}$.

4 Or cette question ne comporte pas qu'un intérêt théorique. Peut-on, en effet, attendre d'un militaire du rang ayant, au cours d'une attaque systématique et/ou généralisée contre une population civile, commis viols et pillages, venir témoigner librement contre son ancien chef de guerre accusé devant une JPI ? Il est raisonnablement permis de douter de la sérénité d'un tel témoin, mais aussi, en conséquence, de la sincérité de sa déposition, en raison de risque de poursuites qu'il encourt dans tous les cas. Mentirait-il à la Cour qu'une peine appropriée viendrait sanctionner son obligation de dire la vérité ${ }^{13}$. Dirait-il enfin cette vérité qu'il serait aussitôt rattrapé par l'histoire et poursuivi, sinon devant la même juridiction, du moins devant les États compétents pour connaître des faits criminels ainsi révélés ${ }^{14}$.

5 La jurisprudence internationale sur le droit au conseil des témoins.- Dans ce contexte, la nécessité, pour cette catégorie de témoins, d'être assistés d'un conseil devenait légitime. Le silence gardé à cet effet par les textes régissant les JPI obligea donc les juges à statuer sur la question. Ainsi, confrontée à la requête d'un témoin de la défense, par ailleurs accusé devant une autre chambre, tendant à voir son conseil l'assister au cours de sa déposition, la Chambre de première III du TPIR dut se pencher sur cette délicate question. Certes, elle fit le constat que "the assistance of a witness by counsel is not the usual practice in common law and civil law systems"15. Elle fit, néanmoins, droit à cette requête en se fondant sur les circonstances exceptionnelles liées au risque d'auto-incrimination ${ }^{16}$. Elle garda la même position devant les requêtes similaires ${ }^{17}$. Cette pratique se répandit par la suite, jusque et y compris devant le TPIY ${ }^{18}$, sans que les juges ne fussent toujours en mesure d'en donner les bases juridiques ${ }^{19}$. 
6 La CPI et le droit au conseil des témoins.- C'est dans ce contexte qu'intervint une décision orale en date du 28 janvier 2009 (ci-après « la Décision orale du 28 janvier 2009 ») rendue par la Chambre de première instance I de la CPI dans l'affaire Lubanga. Aux termes de cette décision, tout témoin susceptible d'auto-incrimination devait se voir systématiquement désigner un conseil ayant principalement pour tâche de lui notifier les garanties de non-incrimination prévues à la règle $74^{20}$. Il s'agissait là d'une avancée significative par rapport aux autres JPI. En effet, d'exceptionnel, le droit au conseil du témoin devint la règle dès la survenance d'un risque d'auto-incrimination. En conséquence, la gravité des faits reprochés au "témoin-suspect» ou "témoinaccusé » n'entrait plus en ligne de compte. De plus, l'intéressé n'était plus tenu, pour pouvoir bénéficier de ce droit, d'en faire la demande à la Chambre. Celle-ci devait désormais lui accorder ce droit, soit d'office, soit à la requête de la partie ou du participant ayant pris l'initiative de la citation du témoin.

7 Conformément à l'article 21-2 du Statut de Rome, qui dispose que «la Cour peut appliquer les principes et règles de droit tels qu'elle les a interprétés dans ses décisions antérieures ", les autres chambres de la Cour s'alignèrent sur la Décision orale du 28 janvier 2009. Aussi, par une décision en date du $1^{\text {er }}$ décembre 2009 (ci-après «l'Instruction 1665 »), la Chambre de première instance II ordonna-t-elle au Greffe « de prendre toutes les mesures nécessaires afin que chaque témoin ne participant pas à la procédure devant la Cour en qualité de victime et susceptible de s'incriminer lors de sa déposition bénéficie d'un avis juridique indépendant fourni par un avocat compétent " ${ }^{21}$. Dans le même ordre d'idées, la Chambre de première instance III prévit, elle, l'hypothèse où les témoins refuseraient de consulter le conseil de permanence qui leur aurait été assigné. Dans ce cas, "leur décision doit être portée à l'attention de la Chambre et de la partie qui les cite $»^{22}$.

8 Problématique.- Pour courageuse qu'elle fut, la Décision orale du 28 janvier 2009 ne tarda guère à montrer ses limites. En effet, en instituant, en l'absence de tout texte de procédure susceptible de l'encadrer, une nouvelle catégorie de conseils chargés d'assister les témoins susceptibles de s'auto-incriminer, cette décision avait laissé la porte ouverte à plusieurs questions dont bon nombre n'ont pas encore trouvé solution à ce jour. Quelle était, en effet, la mission de ce conseil ? Devait-il se limiter à notifier au témoin les garanties de non-incrimination prévues à la règle $74 \mathrm{ou}$, au contraire, assurer la défense des intérêts de son client pendant toute la durée de sa déposition ? Avait-il le droit d'assister son client à l'audience? Bref, jusqu'où pouvait aller un conseil appelé à assister un témoin devant la Cour? La question est d'autant importante que les textes fondamentaux de la Cour ne sauraient nous être d'un quelconque secours, la fonction de conseil du témoin en matière d'auto-incrimination étant de création prétorienne.

9 Méthodologie.- Dans toute démarche scientifique, le recours aux méthodes appropriées permet, non seulement de cerner les différents contours du sujet, mais aussi de tenter de résoudre les problèmes juridiques soulevés dans la problématique. En l'espèce, les méthodes exégétique, comparative et analogique ont été jugées les mieux à même de remplir ce double objectif. Par la méthode exégétique, un examen approfondi des textes fondamentaux de la Cour s'est présentée comme voie obligée pour mieux comprendre la genèse du témoin assisté devant la CPI. Cet examen a permis de relever que cette question n'avait point préoccupé les fondateurs de la Cour, de sorte que le vide juridique existant appelait inéluctablement à s'intéresser au traitement de la 
question devant d'autres JPI. C'est là que la méthode comparative a pris la relève par un rapprochement avec la situation des témoins devant d'autres JPI. Elle a eu le mérite de permettre de mieux apprécier la pertinence des différentes solutions envisagées par les juges pénaux internationaux sur la protection des témoins concernés par la question de l'auto-incrimination. Enfin, la méthode analogique, elle, a permis de mieux comprendre les efforts fournis par les juges de la CPI qui ont su faire preuve de beaucoup d'imagination pour combler les lacunes des textes fondamentaux de la Cour par leur adaptation aux situations inédites qui se présentaient à eux ${ }^{23}$.

Plan du travail.- L'institution du conseil du témoin en matière d'auto-incrimination étant une œuvre prétorienne, c'est dans les précédents mêmes de la Cour qu'il convient de rechercher la mission première de ce conseil, qui est celle de conseil ${ }^{24}$ (chapitre 1). Cependant, au-delà de cette mission primitive, la pratique du témoin assisté devant la Cour a tôt fait de susciter une autre mission, dérivée, elle, de la qualité de défenseur inhérente à la fonction de conseil, à savoir la défense des intérêts de son client (chapitre 2). Il s'agit là, en définitive, des deux piliers de la mission d'avocat. Mais peuton mieux cerner les contours de la mission du conseil du témoin en matière d'autoincrimination sans une connaissance suffisante de ces différentes notions? Un chapitre préliminaire s'avère donc un préalable nécessaire aux analyses qui vont suivre. Une conclusion, qui contiendra des propositions concrètes en vue de l'harmonisation de la mission du conseil du témoin avec les textes fondamentaux de la Cour, viendra clore cette étude.

\section{Chapitre préliminaire Cadre théorique et conceptuel}

11 Plan du chapitre.- Deux concepts-clés, qui se dégagent de l'intitulé de cette étude, méritent d'être brièvement appréhendés avant d'entrer dans le fond du sujet. Il s'agit, d'une part, de la notion même de conseil du témoin (section 1) et, de l'autre, de l'autoincrimination (section 2).

\section{Section $1:$ Le conseil du témoin}

12 Généralités.- Le terme " conseil » peut prêter à confusion, tant il est vrai qu'il est usité dans certaines professions juridiques nationales. Ainsi, en France, avant la réforme de 1990, une nette différence était faite entre avocat et conseil juridique. Depuis, les deux professions ont fusionné, donnant naissance à la nouvelle profession d'avocat, celle de conseil juridique étant supprimée à compter du $1^{\mathrm{er}}$ janvier $1992^{25}$. Il convient donc, avant d'aborder la problématique de l'assistance du témoin devant les JPI (\$.2), de cerner cette notion devant les JPI en général, et la CPI en particulier (\$.1).

\section{§. 1. La notion de conseil devant les JPI}

13 Le conseil devant les JPI.- Le terme « conseil » usité devant les JPI désigne un praticien du droit chargé de représenter une personne, physique ou morale, participant à une procédure pénale internationale. Il est donc équipollent à celui d'avocat employé dans les droits nationaux ${ }^{26}$, c'est-à-dire un " auxiliaire de justice chargé de faire valoir les intérêts d'un défendeur $"^{27}$. Cependant, à la différence des droits nationaux, le monopole de la représentation en justice n'est pas réservé aux avocats devant les JPI. C'est dans ce contexte que le code de déontologie à l'intention des conseils de la défense devant le 
TPIR définit le conseil comme "toute personne considérée par le Greffier comme habilitée à exercer la profession d'avocat dans un État, ou qui est professeur de droit dans une université ${ }^{28}$.

Le conseil à la CPI.- Les textes fondamentaux de la CPI ne définissent pas le mot « conseil ». Il résulte cependant de l'article 17-1 du code de conduite professionnelle des conseils devant cette Cour que ceux-ci sont chargés de prodiguer des avis et de représenter leurs clients devant la Cour. Ils ont donc les mêmes attributions que les conseils prestant devant les autres JPI. L'article $1^{\text {er }}$ de ce code dresse une liste exhaustive des personnes habilitées à porter le titre de conseils, et donc à exercer devant la Cour. Il s'agit des conseils de la défense, des conseils représentant les États, des amici curiae, et des conseils ou mandataires en justice des victimes et des témoins. On peut, de cette énumération, dresser une typologie distinguant les conseils inscrits (I) des conseils non-inscrits (II).

\section{Les conseils inscrits}

15 Typologie.- Les conseils inscrits sont ceux qui figurent sur la liste que tient le Greffier en vertu de la règle 21-2 du RPP. Ils sont, seuls, habilités à représenter les parties et participants intervenant dans le cadre de l'action publique mue devant la Cour ${ }^{29}$. C'est de cette liste que sont désignés les conseils de la défense et les représentants légaux de victimes (A). De la même liste le Greffier dresse un tableau des conseils dont la disponibilité permet une intervention généralement en cas d'urgence : les conseils de permanence (B).

\section{A Les conseils de la défense et les représentants légaux de victimes}

16 Notion.- La représentation des suspects et accusés est assurée par les conseils appelés "conseils de la défense " $^{30}$. Celle des victimes l'est par leurs "représentants légaux». Le terme "victimes» s'entend des personnes, physiques ou morales, ayant subi un préjudice du fait d'un crime dont est saisi la Cour et qui se sont vu reconnaître cette qualité par une chambre ou qui ont introduit une demande en réparation ${ }^{31}$. Le Statut de Rome s'abstient d'utiliser le terme "suspect», lui préférant l'expression, moins

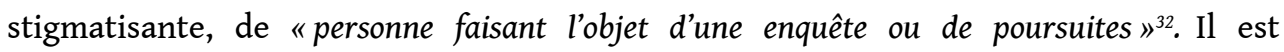
cependant couramment utilisé par les chambres de la Cour $^{33}$, pour désigner une personne contre laquelle "il y a des motifs raisonnables de croire (...) qu'elle a commis un

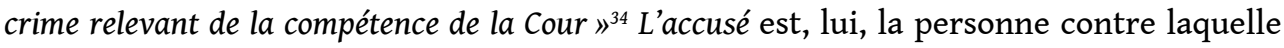
une chambre préliminaire a rendu une décision confirmant les charges retenues contre elle par le Procureur et qui, de ce fait, est renvoyée devant une chambre de première instance pour y être jugée sur les faits qui lui sont reprochés ${ }^{35}$.

Critères de compétence.- Les conseils inscrits étant appelés à officier dans le cadre de la mission principale de la Cour, leur recrutement fait l'objet de critères de sélection très rigoureux. En effet, l'impétrant doit justifier d'"une compétence reconnue en droit international ou en droit pénal et en matière de procédures, et avoir acquis l'expérience nécessaire du procès pénal en exerçant des fonctions de juge, de procureur, d'avocat, ou quelque autre fonction analogue ${ }^{136}$. Le Règlement de la Cour fixe la durée minimale de cette expérience à dix ans ${ }^{37}$.

18 Critère de moralité.- Au-delà des critères de compétence, le candidat sur la liste des conseils doit être d'une moralité exemplaire. Son casier judiciaire, qu'il doit produire, doit être exempt de condamnation pour infractions criminelles ou disciplinaires graves 
considérées comme incompatibles avec la nature des fonctions de conseil devant la Cour $^{38}$. La gravité d'une condamnation et son incompatibilité avec la nature des fonctions de conseil relèvent de l'appréciation du Greffier, sous le contrôle de la Présidence ${ }^{39}$.

La permanence des critères d'admission.- Le conseil inscrit est admis sur base des critères de compétence et de moralité qu'il remplit au moment du dépôt de sa candidature. Il doit, pour pouvoir demeurer inscrit, continuer à remplir les cirières de moralitée ${ }^{40}$. Il a donc l'obligation déontologique de tenir le Greffe informé de tout changement de situation pénale ou disciplinaire de nature à influer sur son maintien sur la liste des conseils ${ }^{41}$. Ainsi, commet une faute disciplinaire, au sens de l'article 31-a du code de conduite professionnelle des conseils, le conseil qui omet délibérément d'informer le Greffier d'une suspension de 12 mois infligée par son barreau d'origine ${ }^{42}$.

Universalité des critères d'admission.- Les critères de compétence et de moralité s'appliquent donc indistinctement à tous les conseils inscrits sur la liste des conseils. Il importe donc peu qu'ils soient conseils de la défense ou représentants légaux de victimes, conseils attitrés ou conseils de permanence.

\section{B Les conseils de permanence}

21 Le tableau des conseils de permanence.- De la liste des conseils, le Greffier tient à jour un tableau des conseils de permanence ${ }^{43}$. Il s'agit des conseils disponibles à tout moment "pour représenter toute personne devant la Cour ou pour se charger des intérêts de la défense" ${ }^{44}$. Le tableau des conseils de permanence est donc issu de la liste des conseils. Il s'ensuit que, forcément, les conseils de permanence remplissent tous les critères de compétence et de moralité exigés des conseils attitrés. La disposition $1^{\text {re }}$ de la norme $74 \mathrm{du}$ Règlement de la Cour qui, dans son écriture du 2 novembre 2011, tient à préciser que les conseils de permanence "doivent avoir au moins dix années d'expérience, telle que définie à la disposition $1^{\text {re }}$ de la norme $67^{\prime \prime}$, constitue donc un rajout dénué d'intérêt. Car les conseils de permanence sont avant tout des conseils inscrits, indépendamment de l'organe qui les désigne.

Organes de désignation des conseils de permanence.

1\% Le Greffe.- Le Greffe désigne les conseils de permanence dans deux cas de figure. Le premier concerne la situation d'une personne bénéficiant du droit au conseil, mais qui, soit ne s'est pas encore vu désigner un conseil attitré, soit n'a pas encore usé du droit qui lui est reconnu de désigner un conseil de son choix. Le deuxième cas de figure est celui d'une personne déjà représentée par un conseil qui se trouve être indisponible. Dans ce cas, le conseil attitré doit consentir à la désignation du conseil de permanence ${ }^{45}$. La mission du conseil de permanence prend fin, soit à la désignation d'un conseil attitré, soit à la fin de l'indisponibilité de ce dernier. Qu'arriverait-il si, tout en étant indisponible, le conseil attitré s'oppose à la désignation d'un conseil de permanence ? La Chambre compétente devra, dans ce cas, décider.

24 2\% La Chambre.- La norme 73-3 du Règlement de la Cour prescrit que «la chambre peut désigner un conseil de permanence en cas d'urgence lorsque le conseil attitré de la personne est indisponible ou lorsque l'intérêt de la justice le commande ». Dans le cas de l'indisponibilité du conseil attitré, il n'est plus fait mention du consentement de ce dernier à la désignation du conseil de permanence. En effet, s'agissant d'un cas d'urgence, la Chambre n'est plus tenue de recueillir l'avis du conseil attitré pour ordonner la 
désignation d'un conseil de permanence. Elle le fera d'ailleurs dans tous les autres cas où elle estimera que l'intérêt de la justice le commande. Le texte ne définit pas l'intérêt de la justice. Il s'agit là des circonstances de fait qui relèvent de l'appréciation souveraine des juges.

Caractère provisoire du statut de conseil de permanence.- Qu'il soit désigné par le Greffe ou la Chambre, le conseil de permanence a de particulier le fait qu'il intervient à titre provisoire, en attendant la désignation d'un conseil attitré. Rien n'empêche cependant le justiciable, au moment de la désignation de son conseil attitré, de porter son choix sur le conseil de permanence dont il aura apprécié les prestations. C'est à la chambre qu'il appartient de rappeler ce droit au justiciable concerné ${ }^{46}$. Dans tous les cas, attitrés ou de permanence, les conseils inscrits sont suffisamment outillés pour représenter les parties et participants intervenant devant la Cour du fait des critères de recrutement rigoureux, lesquels ne sont pas exigés des conseils non-inscrits.

\section{Les conseils non-inscrits}

Notion.- En dehors de la défense, des victimes et de toute personne pouvant bénéficier de l'aide judiciaire de la Cour, les autres protagonistes du procès pénal international autorisés à participer à la procédure sont libres de se faire représenter par des conseils de leur choix. Ceux-ci, dont l'énumération est faite à l'article $1^{\mathrm{er}} \mathrm{du}$ code de conduite professionnelle des conseils (A), ne sont donc pas tenus de figurer sur la liste des conseils de la Cour. Cette affirmation doit cependant être nuancée pour mieux percevoir l'intérêt de la distinction entre conseils inscrits et ceux non-inscrits (B).

\section{A Énumération}

Position du problème.- L'article $1^{\mathrm{er}} \mathrm{du}$ code de conduite professionnelle des conseils devant la Cour énumère les différentes catégories des personnes habilitées à intervenir devant la Cour en qualité de conseils. De cette énumération, on pourrait retenir, comme conseils non-inscrits, les amici curiae et les conseils représentant les États.

Les amici curiae.- L'amicus curiae est un intervenant, volontaire ou sollicité, qui vient apporter sa contribution sur une question de droit de nature à éclairer la religion de la Cour ${ }^{47}$. L'article $1^{\mathrm{er}}$ du code de conduite professionnelle des conseils, dont il découle que tous les amici curiae sans distinction sont qualifiés de conseils, prête à confusion et devrait être nuancé. En effet, la règle 103-1 du RPP, qui traite de la question, dispose qu' "à n'importe quelle phase de la procédure, toute chambre de la Cour peut, si elle le juge souhaitable en l'espèce pour la bonne administration de la justice, inviter ou autoriser tout État, toute organisation ou toute personne à présenter par écrit ou oralement des observations sur toute question qu'elle estime appropriée ». Il faut donc distinguer selon que l'amicus curiae est une personne physique ou morale. Dans la première hypothèse, c'est la personne qui intervient devant la Cour en qualité d'amicus curiae qui sera réputé conseil et, en tant que tel, régi par le code de conduite des conseils devant la Cour si sa demande est admise. Il s'agit, généralement, des personnes ayant des connaissances suffisantes des procédures engagées devant la Cour telles que les avocats ${ }^{48}$ ou les chercheurs ${ }^{49}$.

Dans la seconde hypothèse, il s'agit des personnes morales, c'est-à-dire d'un État ou d'une organisation. Le terme organisation désigne toute association, quelle qu'en soit la nature, qui, de par son objet, détient une certaine expertise sur une question de droit posée à la Cour. Il peut donc s'agir d'une organisation non gouvernementale ${ }^{50}$ ou d'une 
corporation $^{51}$. Dans tous les cas, l'Etat ou l'organisation autorisé à soumettre des observations en qualité d'amicus curiae ne revêt pas la qualité de conseil, mais plutôt la ou les personnes qui le représentent devant la Cour.

Les conseils des États participant à la procédure.- Enfin, les États peuvent être appelés à participer à la procédure à un autre titre que celui d'amici curiae. C'est, notamment, le cas en matière de contestation de la recevabilité d'une affaire ou de la compétence de la Cour. Dans cette hypothèse, l'Etat peut se faire représenter, soit par un agent de l'État ${ }^{52}$, soit par un ou plusieurs conseils ${ }^{53}$. Ceux-ci ne sont nullement tenus de figurer sur la liste des conseils de la Cour. Mais quel intérêt y a-t-il, dans ce contexte, à distinguer les conseils non-inscrits de ceux inscrits?

\section{B Intérêt de la distinction}

Intérêt fondé sur la nature du contentieux.- La différence essentielle entre les conseils inscrits et ceux non-inscrits gît dans la nature du contentieux dans lequel ils interviennent respectivement. En effet, si les conseils inscrits interviennent dans le cadre de l'action publique ${ }^{54}$, qui relève de la compétence principale de la Cour ${ }^{55}$, les conseils non-inscrits interviennent, eux, dans des aspects que l'on pourrait qualifier de secondaires de la procédure pénale internationale. Il s'agit essentiellement des matières d'ordre procédural telles que la contestation de la compétence de la Cour ou de la recevabilité d'une affaire ${ }^{56}$ ou encore sur des questions liées à l'accueil des détenus libérés ${ }^{57}$. Certes, les États peuvent également participer à des procédures de fond, telles que celles relatives à une demande d'asile diligentée par un témoin. Mais il s'agit là des procédures détachables de la procédure principale ${ }^{58}$. Ainsi, pour les rédacteurs du Statut, la charge d'assurer la défense des intérêts des principaux protagonistes de l'action publique devait revenir aux conseils dont les qualifications étaient suffisamment contrôlées, les autres participants étant libres de désigner les conseils de leur choix dans les autres procédures secondaires ou détachables.

Nuance.- Rien n'empêche, cependant, un participant non tenu de choisir son conseil parmi ceux inscrits sur la liste des conseils de la Cour de porter son choix parmi l'un de ces derniers. Un Etat peut donc se faire représenter par un conseil inscrit lors même qu'il n'y serait point tenu. De même, un participant bénéficiant de l'aide judiciaire (suspect, accusé ou victime) n'est pas tenu de choisir son conseil parmi ceux inscrits sur la liste s'il n'a confiance dans aucun d'entre eux. Dans ce cas, il a le droit de recourir à un conseil de son choix, à condition que l'intéressé remplisse les conditions pour être inscrit sur la liste des conseils et consente à y être inscrit. Ce conseil ne pourra effectivement représenter son client qu'après son inscription sur la liste des conseils et après avoir produit une procuration de l'intéressé. Dans l'intervalle, ce dernier sera représenté par un conseil de permanence ${ }^{59}$.

Quid des témoins? - Qu'en est-il des témoins comparaissant devant la Cour ? Jouissentils, eux aussi, du droit au conseil devant cette Cour?

\section{§. 2. La problématique de l'assistance du témoin}

Position $d u$ problème.- Un témoin a-t-il besoin d'être assisté d'un conseil, particulièrement en phase du procès? Une telle question peut paraître singulière lorsqu'on sait que le témoin n'est pas une partie au procès. Il est généralement considéré comme faisant partie des tiers n'ayant qu'un intérêt moral - et non juridique 
- à l'instance ${ }^{60}$. Cette question est différemment réglée par la Cour selon qu'il s'agit des témoins vulnérables (I) ou de ceux susceptibles d'auto-incrimination (II).

\section{Le cas des témoins vulnérables} d'instruction, sans pour autant que ces soupçons soient suffisamment avérés pour justifier une mise en examen. C'est ce qui a fait dire certains auteurs que le témoin assisté est, à la vérité, "un suspect à qui le droit de se défendre est reconnu et un mis en examen potentiel $"{ }^{65}$, que Fabrice Defferrard qualifie, à juste titre, de "para-suspect " ${ }^{66}$. Schématiquement, on peut donc dire qu'au commencement était le témoin, et le témoin devint suspect, et le témoin-suspect eut droit au conseil, devenant ainsi le " témoin assisté ».

41 État de la question en droit international.- Devant les JPI, le droit au conseil du témoin susceptible de s'auto-incriminer est essentiellement prétorien $^{67}$. La pratique est 
cependant exceptionnelle et limitée aux témoins, déjà accusés, sur lesquels pèsent de lourdes charges: "These circumstances include the fact that the potential witness is an accused facing serious charges before this Tribunal, that his testimony might be related to the charges he has to answer, and that the presence of his counsel holding a watching brief may be of great importance in advising him as to his rights set out in Article 20 of the Statute and Rule $90(E) »^{68}$.

Le TSSL, qui a hérité de cette pratique des Tribunaux ad hoc des Nations Unies, l'a assoupli en admettant au bénéfice du droit au conseil même les témoins n'étant pas accusés devant le Tribunal. C'est ainsi que, par une décision en date du 3 août 2010, la Chambre de première instance II autorisa la top-modèle Naomi Campbell, appelée à déposer comme témoin au sujet du diamant que lui aurait offert Charles Taylor lors d'une réception organisée en Afrique du Sud en 1997 par Nelson Mandela, à se faire assister d'un conseil lors de sa déposition : "It is in the interests of justice to grant the right of appearance to one (1) of Ms. Campbell's legal representatives and to grant the said legal representative a limited right to be heard solely on the admissibility of any questions asked of Ms. Campbell which go to possible self-incrimination ${ }^{69}$.

État de la question devant la CPI.- Comme pour les autres JPI, aucun des textes fondamentaux de la Cour ne prévoit le droit au conseil au bénéfice des témoins susceptibles d'auto-incrimination. En effet, il résulte aussi bien de la norme 2-1 du Règlement de la Cour que de la norme 2-1 du Règlement du Greffe que "le terme 'conseil' désigne un conseil de la Défense ou un représentant légal d'une victime». L'article 1-1 de l'Accord sur les privilèges et immunités de la CPI (APIC), adopté par l'Assemblée des États Parties à New York du 3 au 10 septembre 2002, va dans le même sens en disposant : "On entend par 'conseils' les conseils de la défense et les représentants légaux des victimes » L'article 1-v de l'Accord de siège conclu en date du 7 juin 2007 par la CPI et le Royaume des Pays-Bas, entré en vigueur le $1^{\mathrm{er}}$ mars 2008, contient une disposition identique.

La Décision orale du 28 janvier 2009 est donc venue combler une lacune qui a permis à la Cour d'être au même diapason que les autres JPI. Elle a même le mérite d'être en avance dans la mesure où, d'exceptionnel, le droit au conseil du témoin devient la règle dès lors qu'il y a un risque d'auto-incrimination.

\section{Section 2 : L'auto-incrimination}

Notion.- Il y a auto-incrimination lorsqu'un témoin révèle, au cours de sa déposition, des faits susceptibles d'engendrer des poursuites à son encontre. Comme le dit la Chambre de première instance II dans sa décision du $1^{\text {er }}$ décembre 2009 , «il peut régulièrement arriver que des témoins cités à charge ou à décharge se voient poser des questions les obligeant à révéler des informations qui pourraient les incriminer $»^{70}$. Il s'agit donc de la révélation d'une infraction antérieure à la déposition, et non d'une infraction commise devant la Cour comme, par exemple, une atteinte à l'administration de la justice ${ }^{71}$.

La proscription de l'auto-incrimination devant les organes de justice découle du principe général de droit tiré de l'adage «nemo tenetur se ipsum accusare» (\$.1). D’abord appliqué aux suspects, ce principe s'est étendu aux témoins devant les JPI (\$.2.). 


\section{§. 1. Le principe « nemo tenetur se ipsum accusare»} bonne compréhension par ses bénéficiaires pourrait paraître sujette à caution. Aussi, à l'ouverture du premier procès devant la CPI, les juges se sont-ils, avec raison, posé la question de savoir si une notification à l'audience de cette règle aux témoins était suffisante pour leur permettre d'en saisir toute la portée. Leur préoccupation était d'autant légitime que la plupart des témoins attendus étaient des enfants soldats insuffisamment instruits et n'ayant jamais mis les pieds dans une enceinte judiciaire. Aussi jugèrent-ils : "L'application de cette règle 74 doit être expliquée par un juriste qualifié et que cette personne doit bien connaître le cadre et les dispositions du Statut de Rome et l'application du droit pénal en RDC ${ }^{76}$. 
être immédiatement - et j'insiste sur ce terme - immédiatement disponible pour fournir un avis juridique conformément à la règle 74 , alinéa $10 »^{77}$.

Audacieuse, la Décision orale du 28 janvier 2009 l'est, à coup sûr ! D'une part parce qu'elle traduit la volonté des juges de combler les lacunes des textes pour assurer efficacement la protection des droits des témoins-suspects. D'autre part parce qu'on ne peut s'empêcher de constater qu'elle est le fruit d'une gymnastique intellectuelle très compliquée, à la limite de la torsion de la lettre de la règle 74-1 qui est très clair : " À moins que le témoin n'ait reçu l'instruction prévue à la règle 190, la Chambre lui notifie $e^{78}$ les dispositions de la présente règle avant de l'entendre ». Il est, en effet, difficile de considérer un conseil, fut-il de permanence, comme faisant partie de la Chambre. Par ailleurs, il n'est pas dit « la Chambre fait notifier... », encore moins, " un conseil désigné par la Chambre notifie..." ".

Mais qui pourrait se plaindre d'une telle audace lorsqu'on connaît la noblesse de son intention? Bien au contraire, cette décision paraît conforme à l'article 21-3 du Statut de Rome qui dispose que «l'application et l'interprétation du droit prévues au présent article doivent être compatibles avec les droits de l'homme internationalement reconnus ». Le droit au conseil d'un suspect, fut-il par ailleurs un témoin, en est un. C'est certainement la raison pour laquelle les autres chambres de la Cour n'ont pas hésité à emboîter le pas à la Chambre de première instance $\mathrm{I}^{79}$.

Mission du conseil désigné.- La consultation sur les droits prévus à la règle 74 constitue donc la mission primitive, celle de conseil, du conseil ${ }^{80}$ désigné du témoin (chapitre I). Toutes les chambres de la Cour sont unanimes sur cet aspect de la mission du conseil. Cette belle unanimité disparaît cependant dès lors que le fonctionnement de l'institution a tôt fait de faire apparaître le conseil du témoin comme un acteur privilégié chargé d'assurer la défense des intérêts de son client devant lesdites chambres (chapitre II).

\section{Chapitre I La mission primitive : le conseil}

Liminaire.- La mission première assignée au conseil du témoin est d'ordre consultatif : émettre un avis juridique sur la règle 74, c'est-à-dire, en clair, expliquer au témoin le contenu de ce texte. Pour la Chambre de première instance I, qui est à l'origine de cette institution, la nécessité d'un tel avis découle de la complexité même de cette règle : "La règle 74 est une disposition longue et compliquée, et, pour tout témoin (...) il serait extrêmement difficile de comprendre cette disposition et donc, de pouvoir y réagir de manière appropriée" Aussi en a-t-elle tiré comme conséquence que «si cette question d'auto-incrimination apparaissait pendant la déposition d'un témoin, un avocat convenable doit être immédiatement (...) disponible pour fournir un avis juridique conformément à la règle 74 , alinéa $10^{82}$ ". L'avis juridique donné au témoin consiste donc à lui "expliquer les dispositions de la règle $74^{183}$. Il existe donc actuellement une jurisprudence bien établie de la cour sur le rôle consultatif du conseil du témoin sur la règle 74 (section 1). Cependant, si la Chambre de première instance II est demeurée dans la même logique ${ }^{84}$, elle est allée plus loin, jusqu'à considérer que l'avis du conseil pouvait porter sur des questions juridiques autres que celles ayant trait à l'auto-incrimination (section 2). 


\section{Section 1 : Consultation sur la règle 74}

57 Position du problème.- La première préoccupation d'un témoin appelé à déposer devant une chambre de la Cour est de savoir si sa déposition ne peut se retourner contre lui. En d'autres termes, qu'arriverait-il si, en répondant aux questions qui lui sont posées, il révélait la commission des faits susceptibles de déclencher des poursuites à son encontre ? Il n'y a pas de doute que de la réponse à cette préoccupation dépendra la sincérité de la déposition du témoin. En effet, un témoin conscient d'être à l'abri de poursuites déposera avec plus de sérénité qu'un autre qui sera tenté d'orienter sa déposition en guise de défense préventive. La mission principale du conseil d'un tel témoin consiste donc à lui expliquer le droit au silence qui lui est reconnu devant toute question de nature à provoquer une déclaration auto-incriminante (\$.1.). Son attention devra cependant être attirée sur le fait que ce droit n'est pas absolu et que la chambre peut, sous certaines conditions, l'obliger à déposer (\$.2.).

\section{§. 1. Le droit au silence du témoin}

58 Notion.- Le premier droit reconnu au témoin par la règle 74 est celui de " refuser de faire toute déclaration qui risquerait de l'incriminer $»^{85}$. Peu importe, à cet effet, l'auteur de la question. Elle peut, en effet, émaner des parties (accusation, défense) et participants (représentants légaux de victimes) ou même de la chambre elle-même. Il s'agit ici d'un droit exorbitant car faisant exception à l'obligation de témoigner qui s'impose au témoin ${ }^{86}$ sous peine de sanctions pénales pour refus d'obtempérer à un ordre de la Cour $^{87}$.

59 L'exercice de ce droit par le témoin suppose qu'il doit avoir la capacité de détecter à l'avance toute question susceptible de provoquer une déclaration auto-incriminante. Ce qui n'est pas chose aisée lorsqu'on sait que la majorité des témoins appelés à la barre sont des profanes. Il appartient donc au conseil du témoin de lui donner des indices pouvant lui permettre de repérer ce type de questions.

60 La protection du témoin susceptible d'auto-incrimination.- La question relative à la détection des questions auto-incriminantes n'est pas, heureusement, abandonnée au seul témoin concerné. En principe, elle se règle en amont, au moment où une partie ou un participant s'apprête à faire citer un témoin, c'est-à-dire le Procureur ${ }^{88}$, la Défense ${ }^{89}$ et les représentants légaux des victimes ${ }^{90}$. C'est à cette partie ou participant qu'incombe la tâche de faire savoir au Greffe le risque d'auto-incrimination ${ }^{91}$. Si l'entité ayant cité le témoin n'a pas pris cette précaution et que la question apparait en cours d'audience, la chambre suspendra l'audience pour permettre au témoin de se faire assister d'un consei ${ }^{92}$.

61 Le conseil comme dernier rempart contre l'auto-incrimination.- Néanmoins, c'est au témoin lui-même de veiller, à chaque instant, à ne répondre à aucune question de nature à provoquer, de sa part, une déposition auto-incriminante ${ }^{93}$. Et, compte tenu de l'impossibilité, pour le témoin, d'assimiler, à quelques heures de sa comparution, toutes les techniques de détection des questions auto-incriminantes, il a le droit, en cas de doute, d'obtenir une consultation de son conseil pendant la suspension d'audiences ${ }^{94}$. Il peut lui-même demander à tout moment une telle suspension pour les mêmes fins lorsque ce doute lui apparaît en cours d'interrogatoire ${ }^{95}$. Cependant, comme l'exercice de ce droit suppose, de la part du témoin concerné, une capacité d'anticipation que tous 
les témoins ne sauraient avoir, le conseil servira de dernier rempart en demandant à la chambre de suspendre l'audience pour s'entretenir avec son client en pareil cas ${ }^{96}$.

Limites au droit au silence.- Le droit au silence peut avoir des effets pervers, lorsqu'on sait que la preuve testimoniale constitue, on l'a vu ${ }^{97}$, la pierre angulaire du procès pénal international. Que deviendrait, en effet, le procès si la majorité des témoins appelés à la barre se retranchaient derrière ce droit? Aussi s'exerce-t-il sous le contrôle de la chambre qui peut, sous certaines conditions, obliger le témoin à déposer.

\section{§. 2. L'obligation conditionnelle de témoigner}

Note liminaire.- Le droit au silence du témoin n'est pas absolu. Son exercice est, en effet, subordonné au pouvoir dont la chambre dispose en la matière (I), qui peut aller jusqu'à l'anéantir sous certaines conditions (II).

\section{Le pouvoir de la chambre face au droit au silence du témoin}

Face au droit au silence du témoin susceptible de s'auto-incriminer, la chambre dispose d'un pouvoir qu'elle exerce différemment selon que la question posée au témoin est (B) ou non $(A)$ substantielle.

\section{A S'agissant des questions non substantielles}

Notion.- Par question non substantielle, il faudrait entendre celle qui ne porte pas sur un élément constitutif du crime sous examen. A titre d'exemple, pour une chambre saisie d'un crime de guerre d'enrôlement et de conscription d'enfants de moins de 15 ans ${ }^{98}$, une question portant sur des crimes connexes de viol ou d'esclavage sexuel desdits enfants ${ }^{99}$ ne saurait être considérée comme substantielle si la juridiction n'en est pas saisie.

Conséquence.- S'il est posé à un témoin, ancien gardien d'un centre de formation d'enfants soldats, la question de savoir si ces enfants étaient violés ou tenus en esclavage sexuel, il est manifeste qu'il risque fort de s'auto-incriminer s'il avait luimême pris part à ces actes ou les avait facilités de quelque manière que ce soit. Il a donc le droit de refuser de répondre à une telle question. S'agissant d'une question non substantielle, la chambre peut s'incliner devant le refus du témoin et ordonner que l'interrogatoire se poursuive sur des points où l'intéressé ne risque pas de s'autoincriminer ${ }^{100}$. Il en irait autrement si la question à laquelle le témoin est appelé à répondre s'avérait substantielle.

\section{B S'agissant des questions substantielles}

Notion.- Suivant le critère retenu au paragraphe 51 ci-dessus, une question substantielle est celle qui porte sur un élément constitutif du crime dont est saisie la chambre. Elle a donc pour effet d'éclairer directement la chambre sur la responsabilité pénale du suspect ou de l'accusé. Prenons le cas d'un suspect ou accusé poursuivi pour avoir fait participer activement à des hostilités des enfants de moins de 15 ans ${ }^{101}$. Pour étayer l'accusation, le Procureur peut citer à comparaître un ancien commandant du bataillon dans lequel opéraient un ou plusieurs de ces enfants. Une question posée à ce témoin sur l'âge de ses soldats est, à n'en point douter, à considérer comme substantielle, car 
elle a pour objet de permettre à la chambre d'établir directement la responsabilité pénale du suspect ou de l'accusé sur les faits qui lui sont reprochés. déposition (...) resteront confidentiels et ne seront pas révélés au public ou à un État " 103. Cette garantie permet d'éviter que la déposition du témoin ne soit paralysée par la crainte de poursuites éventuelles devant des juridictions nationales à la diligence des particuliers, victimes des crimes dont le témoin pourrait admettre la commission, ou des États compétents pour connaître de tels crimes. Pour qu'une telle garantie ne demeure un vœu pieux, la chambre l'accompagnera des mesures concrètes prévues à la règle 74-7 à savoir :

77 - le huis clos;

78 - la protection de l'identité du témoin et du contenu de sa déposition; 

la chambre préliminaire l'autorisation d'ouvrir une enquête suite à la déposition du témoin. Néanmoins, une requête ultérieure du Procureur tendant à obtenir délivrance d'une citation à comparaître ou d'un mandat d'arrêt contre le même témoin devrait être déclarée irrecevable pour violation de la règle 74-3-c-ii. Seules les personnes autres que le témoin pourront, en effet, faire l'objet de poursuites. Il est manifeste que, éclairé sur cette garantie, le témoin pourrait déposer en toute sérénité, ce qui ne l'empêcherait cependant pas de poser à son conseil d'autres questions juridiques qui lui viendraient à l'esprit.

\section{Section 2 : Consultation sur d'autres questions juridiques}

Liminaire.- Le conseil du témoin ayant été institué pour une question spécifique, à savoir la consultation sur la règle 74 , on pouvait légitimement se demander si une telle consultation pouvait porter sur d'autres problèmes juridiques soulevés par le client. L'on sait, en effet, que la consultation en matière d'auto-incrimination peut engendrer des questions connexes, notamment sur d'éventuelles infractions commises antérieurement à la déposition et dont les poursuites pendant le séjour du témoin à La Haye auraient directement un impact sur sa déposition (\$.1.). Par ailleurs, se trouvant en face d'un conseil, le témoin ne saurait s'empêcher de lui poser toute autre question juridique ayant ou non trait à l'auto-incrimination. Tel est le cas, notamment, des questions portant sur l'étendue des droits reconnus aux témoins comparaissant devant la Cour (\$.2.). Quelle doit être l'attitude du conseil en pareilles circonstances?

La Revue des droits de l'homme, 3 | 2013 


\section{§. 1. De la règle 74 à la consultation générale}

effet d'étendre la mission de conseil du conseil du témoin au-delà de la règle 74 . La Chambre a, par la suite, suffisamment démontré que, pour elle, au-delà de la question d'auto-incrimination, le témoin pouvait obtenir de son conseil «un avis juridique indépendant sur toute autre question $»^{109}$. La consultation pouvait, désormais, porter donc sur toute question juridique posée par le témoin. Dans la pratique, les questions du témoin portent généralement sur ses droits, notamment en matière d'immunités qui lui sont reconnues.

\section{§. 2. La consultation particulière sur les immunités reconnues au témoin}

Position du problème.- En effet, si les garanties de non-incrimination de la règle 74 protègent le témoin des conséquences futures du contenu de sa déposition, il n'en est pas des faits infractionnels non liés à sa déposition. La question, pour les quatre témoins de la défense de Germain Katanga, pouvait bien se poser lorsqu'on sait qu'ils étaient en détention provisoire en RDC pour des crimes contre l'humanité censément commis dans la région de l'Ituri. Ces crimes relevant de la compétence de la Cour, on pouvait craindre que des poursuites fussent engagées contre eux par le Procureur, soit proprio motu ${ }^{110}$, soit sur renvoi de la RDC ${ }^{111}$, pendant leur séjour à La Haye.

La Revue des droits de l'homme, 3 | 2013 
89 Par ailleurs, on peut aussi bien imaginer le cas d'un témoin qui ferait l'objet d'une plainte sur le territoire de l'État hôte pour agression d'un passager ou d'un membre du personnel de l'équipage pendant le vol l'ayant emmené à La Haye. De même en est-il d'un témoin qui se rendrait coupable d'injure publique à l'encontre d'un voisin d'hôtel ou serait impliqué dans une rixe dans une boîte de nuit la veille de sa déposition. Des poursuites engagées contre lui sur le territoire de l'État hôte pour ces faits, pouvant se caractériser notamment par des mesures privatives de liberté, auraient directement pour conséquence de l'empêcher de témoigner devant la Cour. C'est pour éviter ce risque qu'un certain nombre d'assurances, que le conseil est appelé à expliciter à son client, ont été prévues. Ces assurances s'analysent, à la vérité, en immunités qui sont de deux ordres : l'inviolabilité (I) et l'irresponsabilité (II).

\section{L'inviolabilité}

Notion.- L'inviolabilité protège le témoin contre tout risque de poursuites ou de mesures coercitives intempestives avant, pendant et après sa déposition. Elle vise à "garantir aux témoins que leur venue devant la Cour a pour seul objectif de recueillir leur déposition $»^{112}$. Ce type d'immunité est notamment prévu par l'article 93-2 du Statut et l'article 19-1-a de l'APIC. La différence entre ces deux textes gît dans les actes couverts et les modalités d'octroi de la garantie.

91 Les actes couverts. En effet, si l'article 93-2 du Statut couvre les actes commis par le témoin antérieurement à son départ de l'État requis, l'article 19 de l'APIC couvre, lui, tous les faits commis lors des déplacements occasionnés par la comparution (c'est-àdire, en l'espèce, les voyages aller-retour de l'État de résidence du témoin à La Haye, et du quartier pénitentiaire au siège de la Cour), mais aussi pendant et après la déposition.

Les modalités d'octroi des assurances. L'immunité de l'article 93-2 du Statut relève du pouvoir discrétionnaire de la chambre. Elle n'est, dès lors, effective que lorsqu'elle a été accordée par elle, après avis du Procureur et du témoin concerné requis par la règle 191. L'initiative de l'assurance peut provenir, soit de la chambre, soit de la partie qui a appelé le témoin, soit du témoin lui-même. C'est donc au conseil d'informer son client de cette possibilité et, avec son accord, de faire la demande d'assurance à la chambre si aucun des parties et participants n'en a pris l'initiative. Il lui appartiendra, par la suite, de soumettre à la chambre les observations de son client à ce sujet.

En revanche, l'immunité prévue par l'article 19 de l'APIC s'applique de plano, en ce qu'elle ne nécessite pas une décision préalable de la chambre. En tant que disposition d'un accord international, elle lie aussi bien le Procureur de la Cour que les États pouvant se déclarer compétents en vertu de l'article 18 du Statut.

\section{L'irresponsabilité}

Notion. Contrairement à l'inviolabilité, qui laisse subsister l'infraction mais en empêche simplement les poursuites pour les besoins du témoignage, l'irresponsabilité, elle, consiste à rendre le témoin pénalement irresponsable des actes infractionnels commis durant ou à l'occasion de sa déposition. Elle est consacrée par l'article 19-1-c de l'APIC et couvre les paroles, écrits et actes commis pendant et après sa déposition devant la Cour. Il s'agit, selon le texte, d'une «immunité de juridiction totale» qui ne connaît de limite qu'en cas d'atteintes à l'administration de la justice ${ }^{113}$ et d'inconduite à l'audience ${ }^{114}$. 
Effets.- En conséquence, les autorités poursuivantes, en l'occurrence le Procureur de la Cour et les organes judiciaires nationaux, ne sauraient exercer aucune poursuite contre le témoin puisque, par la volonté de l'APIC, celui-ci est rendu pénalement irresponsable des actes infractionnels commis pendant ou à l'occasion de sa déposition. Est-il besoin de préciser qu'une telle immunité de juridiction ne vise qu'à protéger le témoin contre des poursuites de nature à l'empêcher de déposer. Elle ne saurait, dès lors, s'interpréter comme l'empêchant d'exercer ses droits devant les juridictions de l'Etat hôte. Aussi la Chambre de première instance II a-t-elle rejeté comme "sans fondement" le moyen, soulevé par le Greffe ${ }^{115}$, tendant à s'opposer à la demande d'asile introduite auprès des autorités néerlandaises par les témoins détenus de la défense de Germain Katanga en provenance de la $\mathrm{RDC}^{116}$.

Du conseil à la défense.- Si l'Ordonnance du 3 mai 2011 a eu le mérite d'étendre le domaine d'intervention de la consultation du conseil du témoin au-delà de la question de l'auto-incrimination, elle n'est pas sans remettre en question l'essence même de la mission de conseil telle que conçue par la Décision orale du 28 janvier 2009. Qu'arriverait-il, en effet, si la consultation devait déboucher sur la nécessité de l'intervention d'un conseil en faveur du témoin?

Le conseil désigné pourrait-il, sans remettre en cause sa mission primitive de conseil, assurer lui-même la défense des intérêts de son client? La mission de défense du témoin, si tant est qu'elle existe, dérive donc nécessairement de l'exercice de la mission de conseil devant la Cour.

\section{Chapitre II La mission dérivée : la défense}

Position du problème.- La question qui se pose ici est celle de savoir si le conseil du témoin, qui n'a qu'une mission consultative, dispose également d'un mandat de représentation stricto sensu de son client devant la Cour, c'est-à-dire "le pouvoir et le devoir d'accomplir au nom du mandant les actes de la procédure "117, en particulier lorsque que ces actes sortent du contexte de l'auto-incrimination. Au cours d'une conférence de mise en état tenue le 12 mai 2011 par la Chambre de première instance II, le Greffe avait déjà clairement soulevé cette question en soutenant que le conseil des témoins, dont la mission porte sur la règle 74 , n'avait pas compétence pour saisir la Chambre d'une requête en mesures de protection en faveur de ses clients. Car, selon lui, seule la partie ayant cité un témoin était compétente en la matière ${ }^{118}$.

Thèses en présence.- A ce jour, il n'existe pas de réponse uniforme à cette question, les pratiques différant sensiblement d'une chambre à l'autre. On assiste ainsi à une véritable cacophonie jurisprudentielle qui n'est pas près de s'estomper. Car la Chambre d'appel a refusé de connaître des litiges relatifs aux procédures mues par les témoins au motif qu'elles ne relevaient pas de l'appel interlocutoire prévu à l'article $82^{119}$. Un examen attentif des décisions des différentes chambres permet, à ce stade, de relever deux thèses sur cette question : la thèse minimaliste (section 1) et la thèse maximaliste (section 2).

\section{Section 1 : La thèse minimaliste}

Explication.- Pour les tenants de la thèse minimaliste, la mission du conseil du témoin se limite à émettre un avis juridique sur la règle 74 . Il ne peut donc poser un quelconque 
acte de représentation, en particulier lorsque celui-ci n'a pas trait à la question de l'auto-incrimination. Il convient, d'abord, de dresser l'état de cette question devant la Cour (\$.1.) avant d'en faire une appréciation critique (\$.2.).

\section{§.1. Etat de la question}

En soulevant la question relative au mandat du conseil du témoin au cours de la conférence de mise en état du 12 mai 2011 ${ }^{120}$, le Greffe s'était appuyé, en l'absence d'une jurisprudence appropriée, sur celle qui traite du mandat des conseils ad hoc de la Défense (I). Car c'est tout récemment que la Chambre de première instance III a eu à statuer sur le mandat du conseil du témoin en matière d'auto-incrimination (II).

\section{Irrelevance de la jurisprudence sur le mandat du conseil ad hoc de la défense}

2 Position du problème.- L'argument de base de la thèse minimaliste est qu'un conseil désigné pour une mission précise ne peut aller au-delà de son mandat. Par suite, tous les actes qu'il pose et qui ne rentrent pas dans le cadre de son mandat sont considérés comme irrecevables, le Greffe se réservant, dès lors, le droit de ne pas les rémunérer au titre de l'aide judiciaire.

Cas d'application.- Dans le cas sous examen, la Chambre préliminaire I avait, en application de la règle 103, invité Mme Louise Arbour et le Professeur Antonio Cassese, respectivement Haut-Commissaire des Nations Unies aux droits de l'homme et Président de la Commission internationale d'enquête sur le Darfour, à intervenir dans la procédure comme amici curiae sur des questions ayant trait à la protection des victimes et à la préservation des preuves au Darfour ${ }^{121}$. Soucieux de préserver les droits de la défense, la Chambre ordonna, par la même décision, "au Greffier de désigner un conseil ad hoc chargé de représenter et de protéger les intérêts généraux de la Défense dans la situation au Darfour (Soudan) pendant la procédure entamée en application de la règle $103 \mathrm{du}$ Règlement "122. Concrètement, selon la décision de la Chambre, la mission de ce conseil consistait à répondre aux observations présentées par les amici curiae sur des questions ayant trait à la protection des victimes et à la préservation des preuves au Darfour.

4 Par la suite, le conseil ad hoc désigné crut devoir introduire une requête par laquelle il demandait notamment à la Chambre "qu'elle (l')autorise à assister à toutes les procédures prévues par le Statut de Rome, le Règlement de procédure et de preuve et le Règlement de la Cour dans le cadre de la situation au Darfour (Soudan), que ce soit au siège de la Cour ou à l'extérieur, ou en dehors de l'État hôte $»^{123}$. La Chambre rejeta cette requête au motif qu'elle sortait du mandat du conseil ad hoc qui ne portait que sur les procédures relatives à la règle 103 "et ne s'étend pas automatiquement à d'autres procédures au stade préliminaire énoncées dans le Statut et le Règlement " ${ }^{124}$. Elle rejeta également, par voie de conséquence, la demande d'honoraires faite par ce conseil sur la partie des actes supposément posés en dehors de son mandat ${ }^{125}$.

Constats.- $1^{\circ}$ Le premier constat qu'il convient de faire ici est que la décision du 24 juillet 2006 par laquelle la Chambre préliminaire I ordonnait au Greffe de désigner un conseil ad hoc aux fins des procédures relatives à la règle $103 \mathrm{du}$ Règlement de procédure et de preuve n'est pas motivée sur ce point. Toutefois, dans la phase de la situation, la Chambre préliminaire ne pouvait tirer cette prérogative que de l'article 56 du Statut de Rome qui l'investit du pouvoir de protéger les droits de la défense dans la phase de l'enquête. A cet effet, sa mission consiste notamment, «lorsque l'arrestation ou 
la comparution n'a pas encore eu lieu ou que l'avocat n'a pas encore été choisi, à désigner un avocat qui se chargera des intérêts de la défense et les représentera $»^{126}$. On peut donc, à ce stade, légitimement s'interroger sur la capacité de la Chambre à limiter l'intervention de ce conseil à la présentation des observations en réponse à celles soumises par les amici curiae, lors même que le Statut confère à ce conseil un mandat général de protection des intérêts de la défense dans la phase de l'enquête.

$2^{\circ}$ / Bien plus, ce pouvoir de la Chambre préliminaire fait partie des mesures conservatoires qu'elle est habilitée à prendre dans la phase de l'enquête où la défense, à proprement parler, n'existe pas encore. En effet, contrairement à la phase de l'affaire où un suspect est déjà clairement identifié, dans la phase de la situation, il n'y a pas encore de suspect. C'est donc à la Chambre préliminaire qu'il appartient de prendre des mesures conservatoires destinées à sauvegarder, à l'avance, les intérêts de la «partie potentielle (et future) à l'instance pénale ${ }^{127}$.

Différence avec la situation du conseil du témoin.- La situation du conseil ad hoc, agissant en l'absence d'un client de qui il recevrait des instructions précises, n'est donc pas comparable à celle du conseil du témoin. Ce dernier a, en effet, un client présent au siège de la Cour, qui lui demande de poser des actes juridiques sur les droits qui lui sont reconnus par les textes fondamentaux de la Cour, notamment celui de faire une demande de mesures de protection ordinaires ${ }^{128}$ ou spéciales ${ }^{129}$. Quelle attitude doit-il adopter face à une demande aussi précise de son client ? C'est la Chambre de première instance III qui a eu à se pencher sur cette question.

\section{La jurisprudence de la Chambre de première instance III sur le mandat du conseil du témoin}

Origine.- Jugeant attentatoire à sa liberté individuelle les mesures mises en place par l'U.V.T. pour assurer sa protection sur le territoire de l'Etat hôte, un témoin protégé saisit, en date du 19 novembre 2012, la Chambre de première instance III d'une demande de levée desdites mesures par le truchement du conseil de permanence qui lui était désigné dans le cadre de la règle $74^{130}$. Considérant qu'une telle requête allait audelà du mandat du conseil du témoin qui était limité dans le cadre de la règle 74 , la Chambre la rejeta par une décision du 26 novembre 2012 ainsi motivée :

The Chamber reiterates that the mandate of the duty counsel appointed by the Registry to provide notification of the provisions of Rule 74 of the Rules to Witness D04-49 and other witnesses is strictly limited to the issue of self- incrimination. The duty counsel does not represent the witness, is not a party or participant in the proceedings and has no locus standi to appear before the Court. Accordingly, the duty counsel is not entitled to transmit to the Chamber on behalf of the witness any request falling outside the scope of the above mentioned mandate ${ }^{131}$.

110 Jurisprudence de la Chambre sur la question.- A la vérité, cette motivation est une fidèle reproduction de celle contenue dans une décision confidentielle intervenue trois jours plus tôt, laquelle décision rejetait une requête du conseil de permanence tendant à obtenir des mesures de protection en faveur du même témoin ${ }^{132}$. L'intérêt que présentent toutes ces décisions sur l'étendue du mandat du conseil du témoin mérite que l'on s'y penche davantage. 


\section{§.2. Appréciation critique}

111 Par "The Chamber reiterates...", la Chambre entendait s'appuyer sur sa précédente décision confidentielle ${ }^{133}$ qui, elle, s'était, pour délimiter l'étendue de la mission du conseil du témoin, fondée sur le Protocole unique de familiarisation. Il s'agit d'un document élaboré par le Greffe et auquel la Chambre, par une décision du 18 novembre 2010, avait déjà donné force obligatoire dans le cadre du procès qu'elle entendait tenir ${ }^{134}$.

112 Aux termes de ce Protocole, "la Section d'appui aux conseils s'assure qu'un conseil de permanence figurant sur la liste des conseils de la CPI habilités à intervenir dans le cadre de procédures devant la Cour est disponible pour expliquer les dispositions de la (...) règle 74 à tous les témoins qui risquent de s'auto-incriminer et qui ont besoin d'une assistance juridique $»^{135}$. S'agissant du contenu de la consultation, le même Protocole précise, notamment, qu' « il appartient au conseil de permanence d'expliquer au témoin en quoi consiste son mandat ainsi que de l'informer que la discussion portera uniquement sur les questions relatives à la nonincrimination et qu'aucun autre aspect de la déposition ne doit être évoquée $»^{136}$.

113 Nature juridique du Protocole.- Il faudra, d'abord, s'interroger sur la nature juridique de ce protocole et sa force juridique au regard des sources du droit de la Cour. On relèvera, à cet effet, qu'il s'agit d'un document de travail de la Chambre n'ayant aucune valeur normative générale. Il ne saurait donc avoir de force juridique que celle rattachée à la décision de la Chambre qui, si elle peut, en vertu de l'article 21-2, être appliquée par d'autres chambres, ne les lient pas pour autant ${ }^{137}$.

Contrariété de la décision avec le Règlement de la Cour.- $1 \%$ Norme 73-1.- Ensuite, si on ne peut reprocher à la Chambre d'avoir voulu appliquer à la lettre sa propre décision, on s'étonnera, cependant, des extrapolations qui en résultent au point de considérer le conseil de permanence comme ne représentant pas le témoin ou n'ayant point qualité pour comparaitre devant la Cour. En effet, l'affirmation selon laquelle «the duty counsel does not represent the witness " se heurte sérieusement au prescrit de la norme 73-1 du Règlement de la Cour qui fixe la mission du conseil de permanence en ces termes : «Le Greffier recense, dans la liste des conseils, ceux qui acceptent de représenter toute personne devant la Cour ou de se charger des intérêts de la Défense en tant que conseil de permanence ${ }^{138}$. Il s'ensuit que les conseils de permanence devant la Cour ont une double mission : soit intervenir en qualité de conseil ad hoc de la défense, soit représenter toute personne appelée à comparaitre devant la Cour. Il en résulte, en conséquence, que le conseil de permanence désigné pour un témoin est habilité à le représenter devant la Cour.

$1152 \%$ Norme 77-4-f.- Par ailleurs, la norme 77-4-f du Règlement de la Cour prévoit, dans son écriture du 2 novembre 2011, que, s'agissant des témoins cités par la défense, le Bureau du Conseil public pour la défense (BCPD) peut exercer la même mission que le conseil de permanence, c'est-à-dire "assister ou (...) représenter (...) les témoins de la Défense (...) dans les cas prévus à la disposition $1^{\text {re }}$ de la règle 74 , sur instruction ou avec l'autorisation de la chambre ${ }^{139}$. Rappelons, à cet effet, que le mandat de représentation confère au mandataire "le pouvoir et le devoir d'accomplir au nom du mandant les actes de la procédure " ${ }^{140}$. Explicitant ce pouvoir s'agissant des conseils de la défense, la norme 74-2 du Règlement de la Cour pose, notamment, que «lorsqu'elle est représentée par un conseil, la personne ayant droit à se faire assister par un défenseur agit devant la Cour par l'intermédiaire de son conseil, à moins que la chambre n'en décide autrement $»^{141}$. Le conseil de permanence désigné pour un témoin est donc suffisamment qualifié pour poser des actes juridiques 
au nom et pour le compte de son client, notamment en soumettant des requêtes à la chambre.

116 Contrariété de la décision avec la pratique d'autres chambres.- Enfin, contrairement à l'affirmation de la Chambre selon laquelle "(the duty counsel) has no locus standi to appear before the Court", le mandat de représentation du conseil de permanence lui confère, par ailleurs, le pouvoir, s'il échet, de comparaître devant la Cour au nom et pour le compte de son client. En tout cas, c'est ce qu'ont, à juste titre, décidé les autres chambres appliquant la thèse dite maximaliste.

\section{Section 2 : La thèse maximaliste}

Notion.- L'idée générale qui se dégage de la thèse maximaliste est que, dès lors qu'un conseil a été désigné pour un témoin, il devient le seul interlocuteur de la Cour pour toutes les questions liées à sa représentation. Peu importe donc que ces questions soient ou non liées à l'auto-incrimination. On peut trouver une illustration de cette thèse à l'audience du 2 mai 2011 de la Chambre de première instance II, suite à un incident créé par un témoin détenu.

En effet, l'intéressé voulait, en début d'audience, remettre directement aux juges un courrier de demande d'asile adressé aux autorités néerlandaises, avec copies réservées respectivement au président de la Chambre et au Procureur, que l'administration pénitentiaire se refusait à transmettre aux intéressés. Le siège décida qu'il appartenait à ce témoin de remettre son courrier à son conseil qui jugerait de l'opportunité de le transmettre à ses destinataires. Pour la Chambre, en effet, "s'agissant de la correspondance destinée aux autorités du siège de cette Cour, aux juges de cette Cour, il est plus vraisemblable (que le conseil de permanence) estimera qu'il est totalement de son mandat $»^{142}$. Aussi le Président de la Chambre conclut-il à l'intention du témoin: "Vous avez un avocat qui vous a été désigné; ;'est donc important que ce soit lui qui assure cet acheminement " ${ }^{143}$.

Distinction.- Il en résulte que, pour cette Chambre, le conseil de permanence désigné pour assister le témoin dispose d'un mandat de représentation de son client devant elle. A ce titre, il a qualité, non seulement pour poser, au nom et pour le compte de son client, tous les actes de procédure (\$.1.), mais aussi pour comparaître, s'il échet, par devant elle (\$.2.).

\section{§.1. Les actes de procédure du conseil du témoin}

Notion.- Par actes de procédure, il faudrait entendre un ensemble d'écritures que le conseil soumet à la chambre compétente au nom et pour le compte de son client. Ces écritures se présentent généralement sous la forme de requêtes ${ }^{144}$, d'observations ${ }^{145}$ ou de rapports ${ }^{146}$. Institué pour assister le témoin en matière d'auto-incrimination, le conseil du témoin a une compétence indiscutable en la matière (I). Néanmoins, son mandat de représentation lui permet également de poser des actes de procédure même dans les domaines ne relevant pas du contexte d'auto-incrimination (II).

\section{Les actes liés à l'auto-incrimination}

Actes relatifs à la règle 74.- Toutes les chambres s'étant penchées sur la question reconnaissent unanimement au conseil du témoin la compétence de soumettre des 
écritures relatives à la règle 74. Il lui est demandé, à cet effet, d'abord, de confirmer par écrit à la chambre compétente la consultation donnée au témoin ${ }^{147}$. Si, au terme de la consultation, il s'avère que le témoin souhaite obtenir de la chambre les garanties de non-incrimination prévues à la règle 74 , c'est au même conseil qu'il appartiendra d'en faire une demande motivée à la chambre ${ }^{148}$. Ce conseil est donc, en cette matière, habilité à soumettre à la chambre toutes écritures ayant trait à la question d'autoincrimination. Qu'en est-il de celles non liées au contexte d'auto-incrimination?

\section{Les actes non liés à l'auto-incrimination}

Origines.- La brèche ayant permis aux conseils de témoins de poser des actes de procédure non liés à l'auto-incrimination a, sans nul doute, été ouverte par la Chambre de première instance II dont l'Instruction 1665 les a autorisés à consulter au-delà de la règle $74^{149}$. Avant elle, la Chambre de première instance I avait déjà donné l'exemple en permettant, certes en matière d'auto-incrimination, à un conseil de témoin de comparaitre par devant elle ${ }^{150}$. Par la suite, ces deux chambres, mais aussi la Présidence de la Cour, ont reçu des conseils des témoins des requêtes multiples et variées non liées à la question de l'auto-incrimination, auxquelles elles ont donné des réponses appropriées. Il s'agit, pour la plupart, des requêtes en revendication des droits de leurs clients respectifs. Ces requêtes ont donc permis à la Cour d'asseoir une jurisprudence sur les droits des témoins comparaissant devant elle, en particulier les témoins détenus. Elle a donc pu se prononcer, non seulement sur les droits clairement reconnus par les textes fondamentaux de la Cour, mais aussi sur ceux qu'elle a consacrés ellemême, soit en application de l'article 21-3 de son Statut, soit, s'agissant des témoins détenus, par analogie aux suspects et accusés.

Les droits prévus par les textes fondamentaux de la Cour.- Les textes fondamentaux de la Cour reconnaissent aux témoins un certain nombre de droits que ceux-ci peuvent exercer eux-mêmes. Tel est le cas, notamment, du droit à une indemnité de présence ${ }^{151}$, du droit une protection ordinaire $e^{152}$ ou spéciale ${ }^{153}$, ou encore du droit à la vie privée ${ }^{154}$. Ceux des témoins à qui il a été désigné un conseil ne se sont pas privés de bénéficier des services de ce dernier pour revendiquer leurs droits devant les chambres compétentes. Aussi les droits ci-après ont-ils été confirmés par les chambres à la requête des conseils des témoins :

$1^{\circ}$ / le droit, pour un témoin détenu, de bénéficier des avantages pécuniaires au même titre que les témoins en liberté ${ }^{155}$;

$2^{\circ}$ / le droit à la vie privée du témoin, qui implique, pour un témoin détenu ne pouvant regagner son pays d'origine dans un délai raisonnable, celui d'entrer en liaison vidéo avec les membres de sa famille aussi souvent que possible ${ }^{156}$.

Les droits consacrés par la Cour.- L'inexistence d'un texte consacrant un droit aux témoins n'a pas empêché les chambres de les reconnaître en recourant :

$1 \%$ soit à la méthode analogique, auquel cas certains droits, non expressément prévus par les textes fondamentaux de la Cour, ont été, à la requête de leurs conseils respectifs, reconnus aux témoins par analogie à une situation juridique existante. C'est le cas du droit, pour un témoin détenu, non seulement de contester les conditions de sa détention au même titre que les suspects et accusés détenus ${ }^{157}$, mais aussi d'obtenir son transfèrement devant une juridiction de l'Etat hôte statuant sur une requête qu'il lui a soumise ${ }^{158}$. 
$2 \%$ soit à une interprétation extensive des textes existants ou, tout simplement, en ayant recours à l'article 21-3 du Statut de Rome, aux termes duquel «l'application et l'interprétation du droit prévues au présent article doivent être compatibles avec les droits de l'homme internationalement reconnus ». C'est dans ce contexte qu'ont été reconnus :

le droit, pour un témoin malade soigné au quartier pénitentiaire de la Cour, d'obtenir un rapport médical circonstancié sur son état de santé159;

le droit de recevoir communication des pièces de la procédure en vue de préparer sa défense dans une procédure externe à la Cour, en l'occurrence la procédure d'asile devant les autorités de l'Etat hôte ${ }^{160}$;

le droit, pour un témoin détenu dont le statut ne permet d'obtenir une protection efficace de l'U.V.T., de demander l'asile auprès des autorités de l'Etat hôte ${ }^{161}$.

C'est à l'occasion de la reconnaissance de ce dernier droit qu'a été reconnu celui, pour le conseil du témoin, de comparaître devant la Cour.

\section{§.2. La comparution à l'audience}

Liminaire.- La particularité de la fonction de conseil du témoin est que la défense des intérêts de son client se déroule principalement dans le cadre d'une procédure écrite ${ }^{162}$. En revanche, la question se pose s'agissant de l'assistance du témoin à l'audience, car c'est à ce stade de procédure qu'il existe un risque majeur d'auto-incrimination. C'est là certainement le sujet qui divise le plus les chambres de la Cour. On a déjà vu que la Chambre de première instance III excluait toute comparution à l'audience du conseil du témoin ${ }^{163}$. Pour les autres chambres, si, à un moment donné de leur fonctionnement, certaines ont admis une telle comparution, elles l'ont par la suite purement et simplement abandonnée suite aux problèmes qu'elle posait. C'est le cas de la Chambre de première instance I (I). D'autres, en revanche, ont tenté de trouver des palliatifs à ces difficultés, ce qui leur a permis d'autoriser, ou même d'ordonner, le cas échéant, la comparution des conseils des témoins. C'est le cas de la Chambre de première instance II (II).

\section{La pratique de la Chambre de première instance}

Une comparution inédite.- En exécution de la Décision orale du 28 janvier 2009, la Chambre de première instance I autorisa le premier conseil de témoin à comparaitre à son audience du 30 janvier $2009^{164}$. Ce conseil put ainsi assister à la déposition de son client qui se poursuivit jusqu'au 3 février $2009^{165}$. Cette comparution inédite posait cependant deux problèmes majeurs, respectivement relatifs à l'emplacement du conseil et à son rôle à l'audience.

135 L'emplacement du conseil dans la salle d'audience. La configuration des salles d'audiences de la Cour n'ayant pas prévu d'emplacement pour le conseil du témoin, la première question qui se posait était celle de savoir où il fallait placer ce nouveau participant à la procédure. Ceci est d'autant vrai que le pupitre réservé aux témoins n'a été conçu que pour accueillir une personne à la fois. Le conseil ne pouvait donc siéger aux côtés de son client, ce qui eut été sa place naturelle.

La Chambre contourna cette difficulté en installant le conseil à l'emplacement réservé aux représentants des États qui, par bonheur, était inoccupé dans cette phase de procédure. Cet emplacement particulier présentait cependant l'inconvénient d'installer 
le conseil du témoin - qui, en l'occurrence, était un témoin de l'accusation - aux côtés de la défense et en face de l'accusation. Pour un témoin censé charger l'accusé, un tel emplacement était manifestement de nature à le perturber. Le conseil le fit savoir à la Chambre par une écriture du 3 février 2009. La Chambre, qui promit d'y répondre la semaine suivante ${ }^{166}$ ne le fit point.

Rôle du conseil à l'audience. Le deuxième problème que la Chambre de première instance I eut à gérer fut celui relatif au rôle du conseil à l'audience. Devait-il rester muet? Avaitil, au contraire, le droit d'intervenir en faveur de son client, notamment en cas de question de nature à provoquer une déclaration auto-incriminante? Cette question était capitale puisque, de sa réponse dépendait la nécessité ou non de la présence du conseil à l'audience. Elle fut soumise à la Chambre par le conseil du témoin par la même requête du 3 février 2009. Cette requête est, on l'a vu, demeurée sans suite à ce jour.

La fin de la pratique de la comparution des conseils de témoins.- La Chambre se rendit certainement compte que la révolution créée par sa Décision orale du 28 janvier 2009 était de nature à entrainer des conséquences imprévisibles difficiles à gérer. Elle mit donc fin, en attendant sa décision promise sur la requête du conseil de permanence, à la pratique consistant à faire comparaître le conseil du témoin, sa mission s'achevant dès qu'il a fini de notifier à son client la règle 74 . Ce problème inspira la Chambre de première instance II qui réussit à le résoudre autrement.

\section{La pratique de la Chambre de première instance}

Distinction.- Consciente, d'une part, de la nécessité pour le conseil désigné dans le cadre de la règle 74 de suivre intégralement la déposition de son client et d'intervenir, s'il échet, et, d'autre part, de la configuration des salles d'audiences de la Cour qui ne permettait pas une comparution simultanée du témoin et de son conseil à l'audience, la Chambre de première instance II conçut une solution assez originale. Celle-ci distingue, en effet, selon qu'il s'agit d'une simple assistance ou, plutôt, d'une représentation ${ }^{167}$.

cas de l'assistance du témoin. Afin de permettre au conseil du témoin de suivre la déposition de son client et d'intervenir en cas de risque d'auto-incrimination, la Chambre de première instance II fit aménager un bureau équipé d'où le conseil pouvait suivre en temps réel la retransmission de l'intégralité des transcriptions d'audience. Doté d'un logiciel sécurisé de messagerie instantanée, l'ordinateur du conseil du témoin lui permet ainsi d'être relié en permanence avec la salle d'audience et d'intervenir auprès de la Chambre via le greffier d'audience.

Cas de la représentation du témoin.- Saisie d'une requête de trois témoins détenus tendant à obtenir qu'ils soient présentés auprès des autorités néerlandaises pour demander l'asile ${ }^{168}$, la Chambre décida, avant dire droit, de convoquer une conférence de mise en état conformément à la norme 30 du Règlement de la Cour. Il s'agissait, pour elle, notamment d'obtenir des autorités néerlandaises leur avis sur «le statut juridique de ces trois témoins détenus depuis leur arrivée aux Pays-Bas ${ }^{169}$. S'agissant d'une audience destinée à voir débattre des questions techniques, la Chambre décida que les témoins y seraient représentés par leur conseil ${ }^{170}$ qui put ainsi assurer la défense des intérêts de ses clients ${ }^{171}$.

Conclusion 
Constat.- De création prétorienne, la fonction de conseil du témoin concerné par la question de l'auto-incrimination est récente. En l'absence de textes réglementant l'exercice de la mission de cette catégorie de conseils à la Cour, les contours de leur mission ont été - et continuent à être - fixés par les différentes chambres de la Cour, parfois de manière - hélas! - contradictoire. On a ainsi déploré la cacophonie jurisprudentielle existant en la matière, en l'absence du rôle unificateur de la Chambre d'appel qui a refusé de connaître en appel des litiges résultant des procédures engagées par les témoins devant les chambres de première instance ${ }^{172}$.

Nécessité de révision des textes fondamentaux de la Cour.- Il en résulte inévitablement une inégalité de traitement des témoins selon les chambres devant lesquelles ils comparaissent. Seule donc une révision en profondeur des textes fondamentaux de la Cour ${ }^{173}$, pour les mettre en adéquation avec cette nouvelle réalité, serait de nature à aplanir toutes ces discordances. Ces textes devraient clairement confirmer l'institution du conseil du témoin en matière d'auto-incrimination, fixer l'étendue de sa mission et déterminer sa place dans la procédure pénale internationale.

Certes, un amendement du Règlement de la Cour du 2 novembre 2011 tient déjà compte de cette réalité en confiant au BCPD la mission « d'assister ou de représenter (...) les témoins de la Défense (...) dans les cas prévus à la disposition $1^{\text {re }}$ de la règle 74 , sur instruction ou avec l'autorisation de la chambre " "174. Mais cet amendement paraît manifestement incomplet en l'absence des mécanismes de représentation des témoins cités par l'accusation, les victimes ou la chambre.

Exemples à suivre.- Dans le cadre de la révision préconisée, l'avancée de la Cour sur la question de l'auto-incrimination par rapport aux autres $\mathrm{JPI}^{175}$, qu'il convient de saluer, ne devrait cependant pas faire oublier que, sur d'autres sujets liés à la même question, la Cour a encore du chemin à parcourir. Elle devrait donc suivre l'exemple d'autres JPI qui, par la reconnaissance du "right of appearance and audience ", autorisent les conseils de témoins, non seulement à comparaître aux côtés de leurs clients lors de leurs dépositions respectives, mais aussi, sauf lorsqu'ils n'en ont pas fait la demande ${ }^{176}$, à intervenir pendant la déposition de leurs clients sur toute question touchant à l'autoincrimination ${ }^{177}$.

Le prix du respect du droit du témoin à l'assistance d'un conseil.- Certes, la révision souhaitée aurait inévitablement, entre autres, des conséquences procédurales (impact de la présence de participants supplémentaires sur la durée des procédures) et budgétaires (réaménagement des salles d'audiences). Mais c'est là le prix à payer si la Cour tient à être à l'avant-garde de la protection des droits de l'homme par la justice pénale internationale. N'a-t-elle pas jugé, à juste titre d'ailleurs, que «la Cour, expression qui inclut l'ensemble des organes qui la compose, doit appliquer les textes pertinents d'une manière qui soit compatible avec les droits de l'homme internationalement reconnus et exempte de toute discrimination ${ }^{178}$ ? 


\section{NOTES}

1. Sur le procès international en général, lire Liber amicorum Jean-Pierre Cot. Le Procès international, Bruylant, Bruxelles, 2009, 368 p.

2. Lire, à ce sujet, LA ROSA A.-M., «La preuve », in ASCENSIO H., DECAUX E. et PELLET A. (dir.), Droit international pénal, Pedone, $2^{\mathrm{e}}$ éd., Paris, 2012, p. 956 ; NIYUNGEKO G., La Preuve devant les juridictions internationales, Bruylant, Bruxelles, 2005, n 129, p. 137.

3. AMBOS K., "The Right of Non-Self-Incrimination of Witnesses Before the ICC ", LJIL, vol. 15, 2002, p. 173.

4. LA ROSA A.-M., Juridictions pénales internationales. La procédure et la preuve, P.U.F., Paris, 2003, p. 261 ; WIEVIORKA A., Le procès de Nuremberg, L. Levi, Paris, 2006, pp. 64-65.

5. Ch. $1^{\text {re }}$ inst. I, 14 mars 2012, le Procureur c/ Thomas Lubanga Dyilo, Résumé du jugement en application de l'article 74 du Statut, ICC-01/04-01/06-2843-tFRA, par. 10.

6. Le TPIY a été créé par résolution 808 (1993) du Conseil de sécurité des Nations Unies en date du 22 février 1993 (document S/RES/808).

7. Art. 22 et 34 du Statut du TPIY.

8. Art. 34-ii) du RPP du TPIR.

9. La Cour a été mise en place par le Traité adopté à Rome le 17 juillet 1998, Doc. A/CONF.183/9.

10. Art. 43-6 du Statut de Rome.

11. La solution généralement adoptée consiste en ce que la Chambre compétemment saisie peut obliger

un témoin à faire une déposition auto-incriminante, quitte à lui accorder des garanties de nonincrimination pour les faits infractionnels dont il aura révélé la commission (articles 90-E du RPP du TPIY ; 90-E du RPP du TPIR ; 150-F du RPP du Tribunal spécial pour le Liban (TSL) ; 90-E du RPP du Tribunal spécial pour la Sierre Leone (TSSL); et règle 74 du RPP de la CPI).

12. Art. 6-3-c de la Convention européenne des droits de l'homme ; 14-3-d du Pacte international relatif aux droits civils et politiques; 8-2 de la Convention américaine relative aux droits de l'Homme ; 7-c

de la Charte africaine des droits de l'homme et des peuples. Lire, à ce sujet, FORTIN E., "Quatre détenus en République démocratique du Congo entendus à la Cour pénale internationale : saisine inédite établissant la primauté des droits de l'homme internationalement reconnus lors de l'application du Statut de Rome ", Revue québécoise de droit international, vol. 24.2, 2011, p. 58.

13. Art. 70-3 du Statut de Rome ; 91-G du RPP du TPIR ; 91-G du RPP du TPIY ; 152-G du RPP du TSL ; règle 91-C du RPP du TSSL.

14. A titre d'illustration, les articles 211-1, 211-2 et 212-1 à 212-3 du code pénal français prévoient et répriment respectivement le crime de génocide et les autres crimes contre l'humanité. Le Titre I bis

du Livre II du code pénal belge, intitulé «Des violations graves du droit international humanitaire ", prévoit et punit les crimes de génocide, les crimes contre l'humanité et les crimes de guerre. Le titre $\mathrm{V}$ de la loi $\mathrm{n}^{\circ}$ 024/2002 du 18 novembre 2002 portant code pénal militaire de la République démocratique du Congo (RDC) traite, lui aussi, «des crimes de génocide, des crimes contre l'humanité et des crimes de guerre ".

15. TPIR, ch. $1^{\text {re }}$ inst. III, 16 avril 2002, le Procureur c/ André Ntagerura et crts, Decision on Ntagerura's extremely urgent Motion for Order to Transfer an Accused from the Detention Facility in order to Testify for the Defence, pursuant to rules 73 and 54 of the rules of procedure and evidence, ICTR-99-46-T, par. 8.

16. Ibid. 
17. TPIR, ch. $1^{\text {re }}$ inst. III, 17 février 2003, le Procureur c/ André Ntagerura et crts, Decision on Bagambiki's Motion to Transfer an Accused from the ICTR Detention Facility to Testify for the Defence (Pursuant To Rules 73 And 54), ICTR-99-46-T, par. 9.

18. TPIY, 30 août 2010, le Procureur c/ Mičo Stanisić, IT-08-91-T, P.-V. d'audience, p. 13879, 1. 24-25 ; Id., p. 13879, 1. 24-25; TPIY, Ch. $1^{\text {re }}$ inst. II, 12 janvier 2012, le Procureur c/ Zdravko Tolimir, IT-05-88/2-T, P.-V. d'audience, p. 17989, 1. 21-26.

19. TPIY, Ch. $1^{\text {re }}$ inst. I, 12 février 2004, le Procureur c/ Momčilo Krajišnik, IT-00-39-T, P.-V. d'audience, p. 827, 1. 23-25; p. 839, 1. 4-11.

20. Ch. $1^{\text {re }}$ inst. I, 28 janvier 2009, le Procureur c/ Thomas Lubanga Dyilo, Transcription d'audience, ICC-01/04-01/06-T-110-Red3-FRA CT WT, p. 4, 1. 20-23. V., à ce sujet, SHOAMANESH S. S. et MBAYE A.-A., « Article 55. Droits des personnes dans le cadre d'une enquête ", in FERNANDEZ J. et PACREAU X. (dir.), Commentaire article par article du Statut de Rome de la CPI, t. II, Pedone, Paris, 2012, p. 1265.

21. Ch. $1^{\text {re }}$ inst. II, $1^{\text {er }}$ décembre 2009, le Procureur c/ Germain Katanga et Mathieu Ngudjolo Chui, Instructions pour la conduite des débats et les dépositions conformément à la règle 140 , ICC-01/04-01/07-1665-Corr-tFRA, par. 53.

22. Ch. $1^{\text {re }}$ inst. III, 18 novembre 2010, le procureur c/Jean-Pierre Bemba Gombo, Décision relative au protocole unique de préparation et de familiarisation des témoins en vue de la déposition au procès, ICC-01/05-01/08-1016-tFRA, par. 20.

23. V., à titre d'exemple, infra, par. 95.

24. Le terme «conseil » est pris ici dans son sens objectif. Il renvoie donc, non à l'auxiliaire de justice,

mais plutôt à sa fonction qui consiste à conseiller son client.

25. Art. 67 de la loi $\mathrm{n}^{\circ}$ 90-1259 du 31 décembre 1990.

26. L'avocat, comme auxiliaire de justice, est présent dans tous les systèmes juridiques contemporains, même s'il porte un nom différent selon les pays : abogado (Espagne), advogado (Portugal), advokat (Danemark, Norvège, Suède), advokát (Slovaquie, Tchéquie), advocaat (PaysBas, Belgique), advokatas (Lituanie), adwokat-radca prawny (Pologne), anwalt (Allemagne), asianajaja (Finland), avocat (France

et pays francophones), avukat-prokuratur legali (Malte), avvocato (Italie), АДВокАТ (Bulgarie),

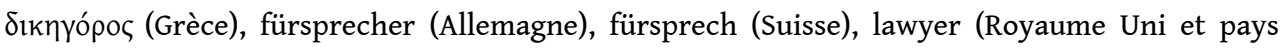
anglophones), lögmann (Island), lögmaður (Island), odvjetnik (Croatie), odvetnik-odvetnica (Slovénie), rechtsanwalt (Allemagne, Autriche, Liechtenstein, Suisse), ügyvéd (Hongrie), vandeadvokaat (Estonie), zverinats advokats (Lettonie).

27. Code de déontologie à l'intention des Conseils de la défense du TPIR, introduction, par. 2. Cette définition est celle d'un conseil de la défense.

28. Art. $1^{\mathrm{er}}$.

29. L'action publique est celle qui est mue dans le cadre de la compétence principale de la Cour, à savoir la connaissance des "crimes les plus graves ayant une portée internationale " (art. 1er du Statut de Rome). Hormis l'Accusation et la Défense, considérés comme les seules parties au procès (V. Ch. app. , 11 juillet 2008, le Procureur c/ Thomas Lubanga Dyilo, Arrêt relatif aux appels interjetés par le Procureur et la Défense contre la Décision relative à la participation des victimes rendue le 18 janvier 2008 par la Chambre de première instance I, ICC-01/04-01/06-1432-tFRA, par. 93, p. 33), les victimes sont les seuls autres protagonistes de l'action publique ayant prérogative de concourir activement

à la manifestation de la vérité, notamment par la production de leurs propres moyens de preuve

(V. MABANGA M. G., La Victime devant la Cour pénale internationale. Partie ou participant?, L'Harmattan, Paris, 2009, pp. 120-122). Les conseils intervenant dans le cadre de cette action sont donc les conseils de la défense et les représentants légaux de victimes.

30. Normes 2-1 du Règlement du Greffe et du Règlement de la Cour. 
31. Lire, à ce sujet, MABANGA M. G., idem, pp. 17-21.

32. V., à ce sujet, HALL C. K., « Article 55 ", in TRIFFTERER O. (ed.), Commentary on the Rome Statute of the International Criminal Court: Obervers' notes, Article by Article, C. H. Beck (München), Hart (Oxford), Nomos (Baden-Baden), $2^{\text {nd }}$ ed., 2008, p. 1097.

33. V., notamment, Ch. prél. I, 29 janvier 2007, le Procureur c/ Thomas Lubanga Dyilo, Décision sur la confirmation des charges, ICC-01/04-01/06-803, pp. 45, 99, 100-104, 125, 127 et 128 ; Ch. prél. I, 30 septembre 2008, le Procureur c/ Germain Katanga et Mathieu Ngudjolo Chui, Décision relative à la confirmation des charges, ICC-01/04-01/07-717-tFRA, pp. 44, 60, 191, 193 et 194 ; Ch. prél. II, 15 juin 2009, le Procureur c/Jean-Pierre Bemba Gombo, Décision rendue en application des alinéas a) et b) de l'article 61-7 du Statut de Rome, relativement aux charges portées par le Procureur à l'encontre de Jean-Pierre Bemba Gombo, ICC-01/05-01/08-424-tFRA, pp. 135, 151, 153, 157, 161, 165 ; Ch. $1^{\text {re }}$ inst. II, 23 janvier 2012, le Procureur c/ William Samoei Ruto et crts, Decision on the Confirmation of Charges Pursuant to Article 61(7)(a) and (b) of the Rome Statute, ICC-01/09-01/11-373, pp. 4, 5, 14, 35, 41 et ss. L'affaire Mbarushimana est certainement celle dans laquelle la Chambre préliminaire a battu

le record de l'usage du mot " suspect », contenu dans 99 pages sur les 237 que compte sa décision refusant de confirmer les charges (Ch. prél. I, 16 décembre 2011, le Procureur c/ Callixte Mbarushimana, Décision relative à la confirmation des charges, ICC-01/04-01/10-465-Red-tFRA).

34. Art. 58-1-a du Statut de Rome.

35. Art. 61-7-a du Statut de Rome.

36. Règle 22-1 du RPP.

37. Norme 67-1.

38. Id.

39. Norme 72-1-d du Règlement de la Cour.

40. On regrettera que le code de conduite professionnelle des conseils ne prévoit pas, à l'endroit des conseils, une obligation de compétence à l'instar du Règlement intérieur du barreau de Paris dont l'article 21.3.1.3 interdit à l'avocat d'accepter «de se charger d'une affaire s'il sait ou devrait savoir qu'il n'a pas la compétence nécessaire pour la traiter, à moins de coopérer avec un avocat ayant cette compétence ".

41. Norme 69-3 du Règlement de la Cour.

42. Com. disc., 9 juillet 2010, Le Greffier c/ M. Hervé Diakiese, Décision du Comité de discipline, DO-01-2010, par. 53.

43. Norme 73-1 du Règlement de la Cour. Si l'amendement du 2 novembre 2011 contient une formulation qui ne reprend pas le mot «tableau », l'idée n'en disparaît pas pour autant. Car en recensant, sur la liste des conseils, ceux acceptant d'intervenir en qualité de conseils de permanence, le Greffier est forcément tenu d'en dresser une liste appelée « tableau ", justement pour éviter toute confusion avec la liste des conseils qu'il tient en vertu de la règle 22-1.

44. Norme 73-1 du Règlement de la Cour.

45. Norme 73-1 du Règlement de la Cour.

46. Ch. prél. I, 20 mars 2006, le procureur c/ Thomas Lubanga Dyilo, Transcription d'audience, ICC-01/04-01/06-T-3-FR, p. 6, 1. 14-21.

47. Pour les détails sur cette question, lire MABANGA M. G., op. cit., pp. 102-103.

48. Ch. $1^{\text {re }}$ inst. I, 15 novembre 2011, le Procureur c/ Thomas Lubanga Dyilo, Order authorising the submission of observations, ICC-01/04-01/06-2821, par. 9.

49. Ch. prél. II, 5 avril 2011, le Procureur c/ William Samoei Ruto et crts, Transmission by the Registry

of the "Request by Ms. Moraa Gesicho to appear as Amicus Curiae », ICC-01/09-01/11-39-Anx.

50. Ch. $1^{\text {re }}$ inst. V, 15 novembre 2012, le Procureur c/ William Samoei Ruto et crts, Decision granting the application by Kituo Cha Sheria for leave to submit observations, ICC-01/09-01/11-473; Ch. prél. II, 17 juillet 2009, le Procureur c/ Jean-Pierre Bemba Gombo, Decision on Request for Leave to 
Submit Amicus Curiae Observations Pursuant to Rule 103 of the Rules of Procedure and Evidence, ICC-01/05-01/08-451, p. 6 ; Ch. prél. I, 18 mai 2012, le Procureur c/ Saif al-Islam Gaddafi et Abdullah al-Senussi, Decision on the Application by Lawyers for Justice in Libya and the Redress Trust for Leave to Submit Observations pursuant to Rule 103 of the Rules of Procedure and Evidence, ICC-01/11-01/11-153, p. 6.

51. Ch. app. , 22 avril 2008, le procureur c/ Thomas Lubanga Dyilo, Decision on « Motion for Leave to File Proposed Amicus Curiae Submission of the International Criminal Bar Pursuant to Rule 103 of theRules of Procedure and Evidence ", ICC-01/04-01/06-1289, par. 8.

52. Ch. app. , 17 août 2011, le Procureur c/ Thomas Lubanga Dyilo, Urgent Request for Directions, ICC-01/04-01/06-2788, p. 5; Id., 21 novembre 2006, Observations de la République démocratique du Congo, ICC-01/04-01/06-720, p. 11; Id., 13 juillet 2011, Application for Leave to Appeal the Trial Chamber's «Decision on the request by DRC-D01-WW0019 for special protective measures relating to his asylum application "

(ICC-01/04-01/06-2766-Conf) dated 4 July 2011, ICC-01/04-01/06-2768, p. 10; Ch. $1^{\text {re }}$ inst. II, 15 juin 2011,

le Procureur c/ Germain Katanga et Mathieu Ngudjolo Chui, Application for Leave to Appeal the Trial Chamber's Decision, ICC-01/04-01/07-3020, p. 8.

ICC-Ol/04-01/07-3003 dated 9 June 2011,

53. Ch. prél. I, 2 octobre 2012, le Procureur c/ Saif Al-Islam Gaddafi et Abdullah Al-Senussi, Libyan Government Request, made in the interests of judicial efficacy, to either : (a) treat the hearing scheduled for 9-10 October 2012 as a status conference; or (b) reschedule the admissibility hearing for November 2012, ICC-01/11-01/11-213, pp. 1, 2 et 12 ; idem, 3 octobre 2012, Decision on the « Libyan Government Request, made in the interest of judicial efficacy, to either : (a) treat the hearing scheduled for 9-10 October 2012 as a status conference; or (b) reschedule the admissibility hearing for November $2012 »$, ICC-01/11-01/11-217.

54. V. supra, par. 13.

55. La CPI est, en effet, d'abord et avant tout une juridiction répressive, dont la mission essentielle

et principale consiste à connaître des "crimes les plus graves ayant une portée internationale " (art. $1^{\text {er }} \mathrm{du}$ Statut de Rome).

56. Art. 19 du Statut de Rome.

57. Norme 51 du Règlement de la Cour.

58. Ch. $1^{\text {re }}$ inst. II, 14 juillet 2011, le Procureur c/ Germain Katanga et Mathieu Ngudjolo Chui, Décision relative à trois demandes d'autorisation d'interjeter appel de la Décision ICC-01/04-01/07-3003 du 9 juin 2011, ICC-01/04-01/07-3073, par. 8.

59. Norme 75-2 du Règlement de la Cour.

60. JOUANNET E., «Incertitudes sur le tiers et désordres de la justice internationale », in RUIZ FABRI H. et SOREL J.-M. (dir.), Le Tiers à l'instance devant les juridictions internationales, Pedone, Paris, 2005, pp. 259 et 261. Dans le même sens, LA ROSA A.-M., Juridictions pénales internationales. La procédure et la preuve, op. cit., pp. 66-67.

61. Règle 88-2 RPP.

62. Supra, par. 3.

63. Art. 113-1 à 113-3 CPP.

64. SOYER J.-C., Droit pénal et procédure pénale, L.G.D.J., 20e éd., Paris, 2008, n 759, p. 311.

65. STEFANI G., LEVASSEUR G. et BOULOC B., Procédure pénale, Dalloz, $22^{\mathrm{e}}$ éd., Paris, 2010, $\mathrm{n}^{\circ}$ 668-1, p. 631.

66. DEFFERRARD F., Le suspect dans le procès pénal, L.G.D.J., Paris, 2005, pp. 26-29.

67. V. supra, par. 5.

68. TPIR, Ch. $1^{\text {re }}$ inst. III, 16 avril 2002, le Procureur c/ André Ntagerura et crts, op. cit., par. 8. 
69. TSSL, Ch. $1^{\text {re }}$ inst. II, 3 août 2010, le Procureur c/ Charles Ghankay Taylor, Decision on Confidential Request for Protective Measures for Naomi Campbell's Testimony, SCSL-03-1-T-1035, p. 6.

70. Instruction 1665 , op. cit., par. 52, pp. 20-21.

71. Article $70 \mathrm{du}$ Statut.

72. Pour approfondir cette question, on lira, avec intérêt, LAZERGES C. (dir.), Figures du parquet, P.U.F., coll. Les Voies du droit, Paris, 2006, 275 p.

73. Lire, à ce sujet, AMBOS K., op. cit., p. 159.

74. Articles 90-E des RPP du TPIY et du TPIR. L'article 150-F du RPP du TSL contient une disposition similaire.

75. Règle 74-5.

76. Décision orale du 28 janvier 2009, op. cit., p. 2., par. 23-25.

77. Id., p. 4, par. 18-21.

78. Le texte d'origine n'est pas souligné.

79. Supra, par. 5.

80. On peut regretter le terme «avocat » utilisé aussi bien dans l'Instruction 1665 que dans la Décision orale du 28 janvier 2009. Le mot «conseil » eut été plus approprié, quoique pouvant créer une confusion entre l'organe (conseil) et sa mission (conseil, par opposition à défense). Car, comme il a été mentionné supra (par. 11 et 12), tous les conseils inscrits sur la liste des conseils de la Cour ne sont pas avocats.

81. Décision orale du 28 janvier 2009, op. cit., p. 2, 1. 17-20.

82. Id., p. 4, 1. 20-23.

83. Ch. $1^{\text {re }}$ inst. III, 7 décembre 2010, le Procureur c/ Jean-Pierre Bemba Gombo, Version modifiée du Protocole unique de préparation et de familiarisation des témoins en vue de leur déposition au procès, présenté le 22 octobre 2010 par l'Unité d'aide aux victimes et aux témoins, ICC-01/05-01/08-1081-Anx-tFRA, par. 57 (ci-après « le Protocole unique de familiarisation »).

84. Instruction 1665, op. cit., par. 53, p. 21.

85. Règle 74-3-a RPP.

86. Règle 65.

87. La Règle 171-4 punit d'une amende de $2.000 €$ toute inconduite à l'audience.

Cette amende peut se renouveler à chaque cas de récidive et est cumulable.

88. Aux termes de l'article 68-1 du Statut, le Procureur est habilité à prendre des mesures de protection

en faveur des victimes et des témoins, en particulier au stade de l'enquête et des poursuites.

89. Ch. $1^{\text {re }}$ inst. II, 13 avril 2011, le Procureur c/ Germain Katanga et Mathieu Ngudjolo Chui, Transcription d'audience, ICC-01/04-01/07-T-246-Red-FRA, p. 2, 1. 7-12.

90. Ch. 1re inst. I, 18 janvier 2008, le procureur c/ Thomas Lubanga Dyilo,

Décision relative à la participation des victimes, ICC-01/04-01/06-1119-tFRA, par. 108, pp. 40-41.

91. Protocole unique de familiarisation, op. cit., par. 56.

92. Règle 74-10.

93. Règle 74-9.

94. Ch. $1^{\text {re }}$ inst. II, 30 mars 2011, le Procureur c/ Germain Katanga et Mathieu Ngudjolo Chui, Transcription d'audience, ICC-01/04-01/07-T-242-CONF-FRA, p. 34, 1. 5-9.

95. Ch. $1^{\text {re }}$ inst. III, 15 octobre 2012, le Procureur c/Jean-Pierre Bemba Gombo,

Transcription d'audience, ICC-01/05-01/08-T-254-CONF-FRA, p. 13, 1. 4-8.

96. Ch. $1^{\text {re }}$ inst. II, 30 mars 2011, op. cit., p. 27, 1. 19-23.

97. Supra, par. 1.

98. Art. 8-2-e-vii du Statut de Rome.

99. Art. 8-2-e-vi du Statut de Rome.

100. Règle 74-6. 
101. Art. 8-2-e-vii du Statut de Rome.

102. Règle 74-5.

103. Règle 74-3-c.

104. La divulgation de l'identité ou de la déposition d'un témoin dans ces circonstances est considérée comme un refus d'obtempérer à un ordre de la Cour et punie d'une suspension de fonctions ne pouvant excéder 30 jours et/ou d'amende inférieure ou égale à $2.000 €$ (règle 71 et art. 171). Une suspension plus longue ne peut, à l'initiative de la Chambre saisie, être prononcée que par la Présidence.

105. SINKONDO M., "Crimes et immunités diplomatiques : Le droit international peut-il à la fois souffler le chaud et le froid?", in CLEMENT G. et LEFEBVRE J. (dir.), Les immunités pénales. Actualités d'une question ancienne, Actes du colloque tenu à Reims le 31 mars 2010, CEPRISCA (Amiens)/ PUF (Paris), 2011, p. 172.

106. Art. 93-2 du Statut de Rome.

107. Ch. $1^{\text {re }}$ inst. II, 3 mai 2011, le Procureur c/ Germain Katanga et Mathieu Ngudjolo Chui,

version publique expurgée de « Ordonnance relative à la mise en œuvre de l'article 93-2 du Statut et des règles 191 et $74 \mathrm{du}$ Règlement de procédure et de preuve au profit de témoins de la défense de Germain Katanga » (ICC-01/04-01/07-2748-Conf), ICC-01/04-01/07-2748-Red, par. 19. Ci-après l' «Ordonnance du 3 mai 2011 ».

108. Id., par. 14.

109. Ch. $1^{\text {re }}$ inst. II, 30 mars 2011, le Procureur c/ Germain Katanga et Mathieu Ngudjolo Chui, Transcription d'audience, p. 21, 1. 21-22 ; Id., 18 avril 2011, p. 20, 1. 17-18 ; Id., 2 mai 2011,

p. 23, 1. 3-5.

110. Art. 15-1.

111. Art. 14.

112. Ordonnance du 3 mai 2011, par. 20.

113. Article 70 du Statut de Rome.

114. Article $71 \mathrm{du}$ Statut de Rome.

115. Ch. $1^{\text {re }}$ inst. II, 12 mai 2011, op. cit., p. 49, 1. 10-14.

116. Ch. $1^{\text {re }}$ inst. II, 9 juin 2011, le Procureur c/ Germain Katanga et Mathieu Ngudjolo Chui, Décision sur une requête en amicus curiae et sur la « requête tendant à obtenir présentations des témoins DRC-D02-P-0350, DRC-D02-P-0236, DRC-D02-P-0228 aux autorités néerlandaises aux fins d'asile » (articles 68 et 93-7 du Statut), ICC-01/04-01/07-3003, par. 74 (Ci-après "la Décision du 9 juin 2011). 117. FRICERO N., «La représentation devant toutes les juridictions », Justice \& Cassation, 2008, p. 90.

118. Ch. $1^{\text {re }}$ Inst. II, 12 mai 2011, op. cit., p. 51, 1. 16-28; p. 52, 1. 1-7.

119. Ch. app. , 26 août 2011, le Procureur c/ Thomas Lubanga Dyilo, Décision relative à la Demande urgente d'instructions présentée par le Royaume des Pays-Bas le 17 août 2011, ICC-01/04-01/06-2837 OA19, par. 8.

120. V. supra, par. 73.

121. Ch. prél. I, 24 juillet 2006, Situation au Darfour (Soudan), Décision invitant à la présentation d'observations en application de la règle $103 \mathrm{du}$ Règlement de procédure et de preuve, ICC-02/05-10-tFR, p. 5.

122. Ibid.

123. Ch. prél. I, 2 février 2007, Situation au Darfour (Soudan), Décision relative à la requête déposée par le conseil ad hoc de la Défense le 18 décembre 2006, ICC-02/05-47-tFR, pp. 3-4.

124. Id., p. 5.

125. Ch. prél. I, 15 mars 2007, Situation au Darfour (Soudan), Décision relative au recours introduit à l'encontre de la décision du Greffe du 13 février 2007, ICC-02/05-66-tFR, pp. 6-7.

126. Art. 56-2-d. 
127. BITTI G., «Commentaire (sur l'intervention de Anne-Marie La Rosa) », in RUIZ FABRI H. et SOREL J.-M. (dir.), Le tiers à l'instance devant les juridictions internationales, Pedone, Paris, 2005, p. 194. Dans le même sens, MABANGA M. G., op. cit., p. 40.

128. Règle 87-1.

129. Règle $88-1$.

130. Ch. $1^{\text {re }}$ inst. III, 19 novembre 2012, le Procureur c/ Jean-Pierre Bemba Gombo, Requête tendant à obtenir levée des mesures coercitives pesant sur le témoin D04-49 (Article 64-6f du Statut de Rome), ICC-01/05-01/08-2416.

131. Ch. $1^{\text {re }}$ inst. III, 26 novembre 2012, le Procureur c/ Jean-Pierre Bemba Gombo, Decision on the duty counsel's « Requête tendant à obtenir levée des mesures coercitives pesant sur le témoin D04-49

(Article 64-6-f du Statut de Rome) », ICC-01/05-01/08-2440, par. 4.

132. Ch. $1^{\text {re }}$ inst. III, 23 novembre 2012, le Procureur c/ Jean-Pierre Bemba Gombo, Decision on the duty counsel's « Demande de mesures de protection en faveur du témoin D04-49 (Règle 87-1 du Règlement de procédure et de preuve) », ICC-01/05-01/08-2436-Conf, par. 6.

133. Supra, par. 83.

134. Ch. $1^{\text {re }}$ inst. III, 10 novembre 2010, op. cit., p. 15.

135. Protocole unique de familiarisation, op. cit., par. 57.

136. Id., par. 63.

137. Le Professeur Robert Kolb considère, avec raison, les décisions de la Cour comme faisant parties

des sources auxiliaires et interprétatives, n'ayant point de caractère obligatoire (KOLB R., Droit international pénal, Bruylant (Bruxelles), Helbing Lichtenhann (Bâle), 2008, pp. 55-60).

138. Le soulignement a été ajouté.

139. Norme 77-4-f du Règlement de la Cour, amendement du 2 novembre 2011, entré en vigueur le 29 juin 2012.

140. FRICERO N., op. cit., p. 90.

141. Le texte d'origine n'est pas souligné.

142. Ch. $1^{\text {re }}$ inst. II, 2 mai 2011, le Procureur c/ Germain Katanga et Mathieu Ngudjolo Chui, Transcription d'audience, ICC-01/04-01/07-T-253-FRA, p. 19, 1. 23-25.

143. Id., p. 20, 1. 2-3.

144. V., notamment, Ch. $1^{\text {re }}$ inst. I, 2 décembre 2011, le Procureur c/ Thomas Lubanga Dyilo, Requête tendant à obtenir production de documents relatifs à l'état de santé du témoin DRC-WW0019, ICC-01/04-01/06-2828-Red; Id., 30 janvier 2009, Transcription d'audience, ICC-01/04-01/06T-113-Red2-FRA CT WT (rev.dec.1974), p. 1, 1. 24-25; Ch. $1^{\text {re }}$ inst. II, 14 mai 2012, le Procureur c/ Germain Katanga et Mathieu Ngudjolo Chui, Requêtes relatives à la détention des témoins DRC-D02P-0236, DRC-D02-P-0228, et DRC-D02-P-0350, ICC-01/04-01/07-3303 ; Id., 31 octobre 2011, Requête aux fins d'autorisation de présenter des observations en qualité d'amicus curiae, présentée par MM. Schüller et Sluiter, conseils du témoin 19 dans le cadre de la procédure de demande d'asile engagée devant les autorités néerlandaises (accompagnée d'annexes), ICC-01/04-01/06-2816tFRA ; Id., 17 octobre 2012, Requête tendant à obtenir transfèrement des témoins DRC-D02P-0236, DRC-D02-P- 0228 et DRC-D02-P-0350 devant la Cour d'appel de La Haye (Article 44-3 de l'Accord de siège), ICC-01/04-01/07-3317.

145. V., à titre d'illustration, Ch. $1^{\text {re }}$ inst. II, 20 juin 2011, le Procureur c/ Germain Katanga et Mathieu Ngudjolo Chui, Observations des témoins DRC-D02-P-0236, DRC-D02-P-0228 et DRC-D02-P-0350 sur les demandes d'autorisation d'appel contre la Décision ICC-01/04-01/07-3003 du 9 juin 2011 (Norme 65-3 du Règlement de la Cour), ICC-01/04-01/07-3028.

146. V., à ce sujet, Ch. $1^{\text {re }}$ inst. II, 30 mars 2011, le Procureur c/ Germain Katanga et Mathieu Ngudjolo Chui, Transcription d'audience, ICC-01/04-01/07-T-242-CONF-FRA, p. 21, 1. 21-25.

147. V., à cet effet, Décision orale du 28 janvier 2009, op. cit., p. 3, 1. 9-11. 
148. Instruction 1665, op. cit., par. 56 ; Protocole unique, op. cit., par. 63.

149. Supra, par. 64 .

150. Infra, par. 96 .

151. Norme 85 du Règlement du Greffe.

152. Règle $87 \mathrm{du}$ RPP.

153. Règle $88 \mathrm{du}$ RPP.

154. Article 68-1 du Statut de Rome.

155. Ch. $1^{\text {re }}$ inst. II, 13 avril 2011, le Procureur c/ Germain Katanga et Mathieu Ngudjolo Chui, Transcription d'audience, ICC-01/04-01/07-T-246-CONF-FRA, p. 4, 1. 1-14.

156. Ch. $1^{\text {re }}$ inst. I, 15 décembre 2011, le Procureur c/ Thomas Lubanga Dyilo, Décision faisant suite aux observations présentées par les conseils représentant le témoin 19 de la Défense dans le cadre de la procédure de demande d'asile engagée devant les autorités néerlandaises, ICC-01/04-01/06-2835-tFRA, par. 22; La Présidence, 18 décembre 2012, Decision on the application for judicial review dated 5 November 2012, ICC-RoR221-04/12-4-Red, par. 31.

157. Ch. $1^{\text {re }}$ inst. II, 15 juillet 2011, le Procureur c/ Germain Katanga et Mathieu Ngudjolo Chui, Ordonnance relative aux conditions de détention des témoins détenus, ICC-01/04-01/07-3078.

158. Id., 7 septembre 2012, Ordonnance portant sur la requête du conseil de permanence relative au transfèrement des témoins DRC-D02-P-0236, DRC-D02-P-0228, et DRC-D02-P-0350 devant la Cour de district de La Haye (Article 44-3 de l'Accord de siège), ICC-01/04-01/07-3314, par. 7.

159. Ch. $1^{\text {re }}$ inst. I, 7 décembre 2011, le Procureur c/ Thomas Lubanga Dyilo, Order on the medical reports relating to defence Witness 19 , ICC-01/04-01/06-2830, par. 11.

160. Ch. $1^{\text {re }}$ inst. II, $1^{\text {er }}$ juin 2012, le Procureur c/ Germain Katanga et Mathieu Ngudjolo Chui, Ordonnance relative aux requêtes du conseil de permanence relatives à la détention des témoins DRC-D02-P-0236, DRC-D02-P-0228, et DRC-D02-P-0350, ICC-01/04-01/07-3303, par. 9-10.

161. Décision du 9 juin 2011, op. cit., 73 ; Ch. $1^{\text {re }}$ inst. I, 5 août 2011, le Procureur c/ Thomas Lubanga Dyilo, Version publique expurgée de la Décision relative à la requête de DRC-D01-WW0019 aux fins de mesures de protection spéciales dans le cadre de sa demande désile, ICC-01/04-01/06-2766-Red-tFRA, par. 83 et 84.

162. V. supra, par. 90.

163. V. supra, par. 82.

164. Ch. $1^{\text {re }}$ inst. I, 30 janvier 2009, le procureur c/ Thomas Lubanga Dyilo, Transcription d'audience, ICC-01/04-01/06-T-113-Red2-FRA CT WT (rev.dec.1974), p. 1, 1. 12-17.

165. Ch. $1^{\text {re }}$ inst.. I, 3 février 2009, le procureur c/ Thomas Lubanga Dyilo, Transcription d'audience, ICC-01/04-01/06-T-114-FRA.

166. Ch. $1^{\text {re }}$ inst. I, 6 février 2009, le procureur c/ Thomas Lubanga Dyilo, Transcription d'audience, ICC-01/04-01/06-T-120-FRA, p. 2, 1. 15-18.

167. Pour une distinction entre les deux concepts, on lira, avec intérêt, FRICERO N., op. cit., pp. 90-91.

168. V. supra, par. 94.

169. Ordonnance du 5 mai 2011, op. cit., par. 14.

170. Ordonnance du 5 mai 2011, op. cit., p. 8 ; Ch. $1^{\text {re }}$ inst. II, 12 mai 2011, le Procureur c/Germain Katanga et Mathieu Ngudjolo Chui, Transcription d'audience, ICC-01/04-01/07-T-258-FRA, p. 2, 1. 7-8.

171. Id., pp. 7-29.

172. Supra, par. 74 .

173. Particulièrement le Statut, le RPP ainsi que le Règlement de la Cour.

174. V. supra, par. 87.

175. Supra, par. 5 . 
176. TSSL, 3 août 2010, le Procureur c/ Charles Ghankay Taylor, Decision on motion for right to appear as counsel to a witness, SCSL-03-01-T-1042, p. 3.

177. V., sur cette question, TSSL, Ch. $1^{\text {re }}$ inst. II, 3 août 2010 , op. cit., p. 4 ; TPIR, ch. $1^{\text {re }}$ inst. III, 17 février 2003, op. cit., par. 10.

178. Décision du 9 juin 2011, op. cit., par. 77.

\section{AUTEUR}

\section{GHISLAIN MABANGA}

Avocat au barreau de Paris, conseil à la Cour pénale internationale et chargé d'enseignements à l'Université Paris Ouest - Nanterre La Défense. 